\title{
On Transversely Isotropic Elastic Media with Ellipsoidal Slowness Surfaces
}

\author{
Anna L. Mazzucato ${ }^{\mathrm{a}, *, 1}$, Lizabeth V. Rachele ${ }^{\mathrm{b}, 2}$ \\ a Department of Mathematics, Pennsylvania State University, University Park, PA 16802, USA \\ binverse Problems Center, Rensselaer Polytechnic Institute, Troy, New York 12180, USA
}

\begin{abstract}
We consider inhomogeneous elastic media with ellipsoidal slowness surfaces. We describe all classes of transversely isotropic media for which the sheets associated to each wave mode are ellipsoids. These media have the property that elastic waves in each mode propagate along geodesic segments of certain Riemannian metrics. In particular, we study the intersection of the sheets of the slowness surface. In view of applications to the analysis of propagation of singularities along rays, we give pointwise conditions that guarantee that the sheet of the slowness surface corresponding to a given wave mode is disjoint from the others. We also investigate the smoothness of the associated polarization vectors as functions of position and direction. We employ coordinate and frame-independent methods, suitable to the study of the dynamic inverse boundary problem in elasticity.
\end{abstract}

Key words: Elastodynamics, transverse isotropy, anisotropy, ellipsoidal slowness surface $2000 M S C: 74 \mathrm{Bxx}, 35 \mathrm{Lxx}$

\section{Introduction and Main Results}

In this paper we consider inhomogeneous, transversely isotropic elastic media for which the associated slowness surface has ellipsoidal sheets. Transverse isotropy is characterized by the property that at each point the elastic response of the medium is isotropic in the plane orthogonal to the so-called fiber direction. We consider media in which the fiber direction may vary smoothly from point to point. Examples of transversely isotropic elastic media include hexagonal crystals and biological tissue, such as muscle.

Deformations in elastic media are described by solutions of a $3 \times 3$ system of partial differential equations. We represent the elastic body by a bounded region $\Omega \subset \mathbb{R}^{3}$, and we assume that its boundary is smooth. In the small deformation regime this system reduces to the following linear, nonconstant-coefficient system:

$$
\left(P_{\rho, \mathbf{C}} \mathbf{u}\right)_{i}=\rho(x) \frac{\partial^{2} u_{i}}{\partial t^{2}} \quad-\sum_{j, k, l=1}^{3} \frac{\partial}{\partial x_{j}}\left(C_{i j k l}(x) \frac{\partial u_{k}}{\partial x_{l}}\right)=0, \quad x \in \Omega, \quad t \geqslant 0, \quad i=1,2,3,
$$

\footnotetext{
* Corresponding author.

Email addresses: mazzucat@math.psu.edu (Anna L. Mazzucato), rachel@rpi.edu (Lizabeth V. Rachele).

1 The first author was partially supported by NSF grant DMS-0405803.

2 The second author was supported by NSF grant DMS-0340530.
} 
where the density and elastic parameters are modeled by the $C^{\infty}(\bar{\Omega})$-smooth function $\rho$ and rank-4 tensor field $\mathbf{C}$, and the vector-valued function $\mathbf{u}(x, t)$ gives the displacement after time $t$ of a point initially at position $x=\left(x_{1}, x_{2}, x_{3}\right)$. (See Section 2.1.)

Slowness surfaces arise naturally in the study of elastic wave propagation. In the case of constant density $\rho$ and elasticity $\mathbf{C}$, a plane wave with wave normal $\mathbf{n}$ will be a solution of (1) if the speed $c(\mathbf{n})$ of the front with normal $\mathbf{n}$ satisfies a certain characteristic condition. When the coefficients of (1) are not constant, plane waves do not generally satisfy (1), but any solution can be approximated locally near a point as a superposition of plane waves. The characteristic condition is given by

$$
\operatorname{det}\left|-\rho \delta_{i k}+\mathrm{C}_{i j k l} s_{j} s_{l}\right|=0
$$

in terms of the slowness variable $\mathbf{s}=\mathbf{n} / c(\mathbf{n})=\boldsymbol{\xi} / \tau$, where $\tau, \boldsymbol{\xi}$ are dual variables to $t, x$. (Here, and throughout the paper, we use the convention of summation over repeated indices, and we generally omit the explicit dependence on $x$.) Equation (2) defines the degree-6 slowness surface as the locus of points $(x, \mathbf{s})$ where the principal symbol $-\rho \tau^{2} \delta_{i k}+\mathrm{C}_{i j k l} \xi_{j} \xi_{l}$ of $P_{\rho, \mathbf{C}}$ is not invertible. The $m$-th eigenvalue $\rho\left[-\tau^{2}+c_{m}(x, \boldsymbol{\xi})\right]$ of the principal symbol is homogeneous of order 2 in $\boldsymbol{\xi}$, and so the slowness surface consists of the three sheets $\tau^{2}=c_{m}^{2}(\boldsymbol{\xi})$, that is, $c_{m}^{2}(\mathbf{s})=1$. We write $\mathbf{v}_{m}(\mathbf{s}), m=1,2,3$, for the three non-colinear eigenvectors, called the polarization vectors. The $m$ th family of waves, or $m$ th-wave modes, are disturbances propagating along characteristics corresponding to the $m$ th-eigenvalue. To first order the speed of propagation of the wave front of the $m$ th mode is given by $c_{m}(\mathbf{s}),|\mathbf{s}|=1, m=1,2,3$, and the direction of displacement for the $m$ th mode is given by the polarization vector $\mathbf{v}_{m}(\mathbf{s})$. In isotropic elastic media there are two elastic wave modes, the shear and compressional modes. We refer, for instance, to Musgrave [1] for a discussion of wave propagation in crystals.

In this paper we study the slowness surfaces and polarization vectors for certain classes of transversely isotropic media. Our main motivation is the microlocal analysis of propagation of singularities along rays for the system (1). In [2] we apply this analysis to the inverse problem of determining the elastic moduli in the interior from dynamic displacement-to-traction measurements made at the surface. In these applications the interior of the elastic body is generally not directly accessible, and it is important to consider inhomogeneous materials. For these reasons we work in a coordinate and frame-independent fashion.

At each point $x$, sheets of the slowness surface are described by homogeneous equations of degree two in the slowness variable $\mathbf{s}$. When each of the sheets is ellipsoidal, it can be written in the especially simple form $1=$ $\mathbf{s}^{t} \boldsymbol{G}^{-1}(x) \mathbf{s}$, with $\boldsymbol{G}(x)$ a symmetric, positive-definite matrix. If this is the case for all points $x \in \Omega$, rays along which each wave family propagates are (piecewise) geodesic segments with respect to the Riemannian metrics $\boldsymbol{G}$, and the mathematical description of wave propagation is considerably simplified. More generally, rays are integral curves of Hamiltonian functions, which, when the Hamiltonian is regular, are geodesics of Finsler metrics [3, Chapter 1, Section 1], [4, Section 1.1], [5].

From the point of view of the inverse problem we distinguish the case in which the slowness surface is a union of ellipsoids from the case in which each sheet $c_{m}^{2}(\mathbf{s})=\mathbf{1}$ is an ellipsoid. In the first case wave propagation may be described in terms of Riemannian metrics, but rays can change from one mode to another at some intersection points of the sheets of the slowness surface, while in the second case rays may be described as geodesics of the Riemannian metric $\boldsymbol{G}_{m}$ associated with a particular mode $c_{m}^{2}(s)=1$, and rays do not change mode. This observation motivates our detailed study of the intersections of the sheets of the slowness surface. Media with ellipsoidal slowness surfaces are referred to as ellipsoidal media, while those with ellipsoidal sheets of the slowness surface we refer to as having geodesic wave propagation $(G W P)$. We make this distinction, because in the case of $(G W P)$ the metrics $\boldsymbol{G}_{m}, m=1,2,3$, can be recovered from information about the propagation of singularities in the solution of the system (1), and each of them in turn carries information about the material parameters $\rho, \mathbf{C}$.

In this work we present all possible classes of transversely isotropic elastic media with geodesic wave propagation: two families denoted by (GWP1) and (GWP2), parametrized by 4 elasticity parameters, together with the density $\rho$ in the dynamic case, and two parameters for the fiber direction $\mathbf{k}(x)$ which we allow to vary from point to point. General transversely isotropic media are described by five elasticity parameters.

Classes of elastic media similar to (GWP1) and (GWP2), denoted here by (Q1) and (Q2), for which the slowness surface is the union of ellipsoids, have been studied by many authors. The conditions $(Q 1)$ and $(Q 2)$ are introduced 
by Rudzki [6], and studied by Helbig [7,8], Payton [9], [10], Chadwick [11], Chadwick and Norris [12, (1.1),(1.2)], Burridge, Chadwick, and Norris, [13, (3.13),(6.13)], and Bakker [14], for example. The results in these papers are in terms of a special basis relative to the fiber direction. Here we consider a general basis. While (GWP2) and (Q2) coincide, $(G W P 1)$ is a proper subclass of $(Q 1)$. There is one additional class [12, (1.3)] of anisotropic elastic media for which all three modes are ellipsoidal, a case of orthotropic elasticity.

General transversely isotropic elastic media have been studied by comparing with those having ellipsoidal slowness surfaces. In fact, Chadwick [11] considers transversely isotropic elastic media that are inextensible in the fiber direction via a limiting process from a space of transversely isotropic elastic media with ellipsoidal slowness surfaces; see also Daley and Lines [15].

In the case of elliptic, homogeneous transverse isotropy the analysis of the system (1) can be simplified by decoupling the system into linear, scalar wave equations for each of the wave modes. To this end Burridge, Chadwick, and Norris [13] present the Green's tensor on $\mathbb{R}^{3}$ for this case in terms of fundamental solutions of these scalar wave equations.

We begin this work by deriving the conditions $(Q 1) c_{13}+c_{44}=0$ and (Q2) $\left(c_{13}+c_{44}\right)^{2}=\left(c_{11}-c_{44}\right)\left(c_{33}-c_{44}\right)$ in Section 4. These conditions characterize transversely isotropic elastic media with sheets of the slowness surface of the form $1=c^{2}(\mathbf{s})=p \pm|q|$, where $p(\mathbf{s}), q(\mathbf{s})$ are homogeneous polynomials of degree 2 in $\mathbf{s}$. In the case $(Q 1)$ we obtain new conditions $(G W P 1)$ in Section 4. The conditions $(G W P 1)$ and $(Q 2)=(G W P 2)$ guarantee that the sheets $1=c^{2}(\mathbf{s})$ of the slowness surface are given in terms of polynomials $c^{2}(\mathbf{s})$ in $\mathbf{s}$. In particular, the eigenvalues of the principal symbol of $P_{\rho, \mathrm{C}}$ are smooth functions of $x$ and $\xi$, a condition important in microlocal analysis.

Our derivation is based on Theocaris and Philippidis' [16] spectral representation of the transversely isotropic elasticity tensor, and is independent of a choice of frame for the tangent bundle. Working in a frame-invariant setting is natural in studying elastodynamics from a differential-geometric point of view. We employ this point of view in [17], where we consider the pullback of an elasticity tensor, and study the type of anisotropy that occurs in the orbits of anisotropic elasticity tensors under the action of pullback by a diffeomorphism, and in [2], where we show that the travel time of a wave mode through an anisotropic elastic object is determined by traction-displacement surface measurements in the case that the corresponding sheet of the slowness surface is disjoint from the others. For transversely isotropic elastic media with slowness surfaces having ellipsoidal sheets, we then apply the results of this paper to conclude that two of the elasticity parameters are partially determined by dynamic surface measurements. Our methods are especially suited to treat non-homogeneous materials.

The disjoint mode condition is not restrictive from the point of view of actual physical media. By a simple computation (using Maple) we find that each of the 204 transversely isotropic elastic materials listed in [18, pages 3949] has a disjoint mode, and two (a form of the ceramic $\mathrm{BaTiO}_{3}$ with $\mu_{L}=43, \mu_{T}=44.5, A=121.5, B=162, C=78$, and a polymer polymethyl methacrylate (Perspex) with $\mu_{L}=2.3, \mu_{T}=2.1, A=6.0, B=11.0, C=4.8$ ) are very close to $(G W P)$. These two materials are (essentially) of type (GWP2).

To prove the uniqueness of the travel times of the disjoint mode in general anisotropic media, we apply techniques from microlocal analysis to study wave propagation. Microlocal analysis of wave propagation may be seen as an extension of the classical ray-tracing approach well known in seismic exploration. From that point of view an elastic medium has GWP if the determinant of the principal symbol of the operator $P_{\rho, \mathbf{C}}$ in (1), i.e., the determinant of $\rho \tau^{2} I-\mathbf{Q}(\mathbf{n})|\boldsymbol{\xi}|^{2}$, where $\mathbf{Q}(\mathbf{n})$ is the acoustical tensor, may be factored at each $x \in \Omega$ as

$$
-\rho^{3}\left(\tau^{2}-\boldsymbol{\xi}^{t} \boldsymbol{G}_{1}^{-1} \boldsymbol{\xi}\right)\left(\tau^{2}-\boldsymbol{\xi}^{t} \boldsymbol{G}_{2}^{-1} \boldsymbol{\xi}\right)\left(\tau^{2}-\boldsymbol{\xi}^{t} \boldsymbol{G}_{3}^{-1} \boldsymbol{\xi}\right),
$$

where the $\boldsymbol{G}_{i}$ are smooth, symmetric, positive-definite matrices depending only on the position $x$. The principal symbol is formally obtained from $P_{\rho, \mathbf{C}}$ by replacing $D_{t}=-i \partial_{t}$ with the frequency $\tau$, and each partial derivative $D_{j}=-i \partial_{x_{j}}, \quad j=1,2,3$, with the $j$ th-component of the dual vector $\boldsymbol{\xi}$. The three eigenvectors $\mathbf{v}_{i}=\mathbf{v}_{i}(\boldsymbol{\xi})$ of the principal symbol are the polarization vectors introduced before. The wave normal $\mathbf{n}$ corresponds to the dual vector $\boldsymbol{\xi}$ divided by its length.

Microlocal analysis seeks approximations to solutions to partial differential equations, such as (1), in the form of oscillatory integrals or superpositions of waves with nonconstant amplitude and phase. For each wave mode, the phase function satisfies an eikonal equation, a non-linear partial differential equation that can be directly obtained from the equation describing each sheet of the slowness surface. We derive the slowness surfaces (and so the eikonal 
equations) for transversely isotropic media with GWP in Section 4. (For an introduction to microlocal analysis and oscillatory integrals, we refer for example to the monograph [19].)

We derive mild conditions on the material parameters in Corollaries 2 and 3 ensuring that the light cone (or the sheet of the slowness surface) associated with one wave mode is always disjoint from the others. In these cases the corresponding eigenvector (or polarization vector) is smooth as a function of position $x$ and direction $\boldsymbol{\xi}$. In [2] we observe that since the eigenvector associated with the disjoint mode is smooth everywhere, then, informally, the operator $P_{\rho, \mathbf{C}}$ in (1) can be (partially) factored and the analysis of the component for that particular wave mode reduces to the analysis of a scalar differential operator. When the sheet of the slowness surface associated with the disjoint mode is ellipsoidal, this operator gives rise to an anisotropic wave equation of the form

$$
\partial_{t t} \mathbf{u}-\Delta_{G} \mathbf{u}+L \mathbf{u}=0
$$

where $\boldsymbol{G}$ is one of the metrics $\boldsymbol{G}_{i}$ appearing in the factorization (3), $L$ is a first-order operator, and $\Delta_{G} \mathbf{u}$ is given by $G^{k l} \partial_{x_{k}} \partial_{x_{l}} \mathbf{u}$, plus lower-order terms. When the slowness surface is a union of ellipsoids, but the media is not $(G W P)$, e.g. in the class $(Q 1) \backslash(G W P 1)$, this reduction does not apply.

Smoothness of an eigenvector can fail if the multiplicity of the corresponding eigenvalue changes, that is, if two distinct sheets of the slowness surface intersect. We prove that, for transversely isotropic media with GWP, two of the three sheets always intersect, and there are only two types of intersection: (1) tangential intersection along the fiber direction $\mathbf{k}$, or (2) coincident modes, i.e., two of the three wave modes agree everywhere. Coincident modes occur, for example, in isotropic elastic media, but in Section 4 we present a case of transversely isotropic media with coincident modes which does not reduce to isotropic.

The paper is organized as follows. We discuss the equations for elastodynamics and introduce the spectral representation for the transversely isotropic elasticity tensor in Section 2; we define certain convenient combinations $A, B, C$ of the material parameters and describe the sheets of the slowness surface for general transversely isotropic elastic media in Section 3; and we derive the conditions $(Q 1),(Q 2)=(G W P 2)$, and $(G W P 1)$ that guarantee ellipsoidal slowness surfaces in Section 4. In Section 5 we give conditions under which any two of the sheets of the slowness surface intersect, and we describe conditions under which the type of intersection changes by classifying all positive-definite, transversely isotropic elastic media in terms of the so-called anellipticity parameter $a_{C}=C+\mu_{L}$, where $\mu_{L}$ is the longitudinal shear modulus. In Section 6 we describe when strongly elliptic, ellipsoidal, transversely isotropic elastic media have a disjoint sheet of the slowness surface, and we study the polarization vectors for transversely isotropic elastodynamics with $(G W P)$. Finally, we collect some of the more technical proofs and derivations in Section 7 .

\section{Preliminaries}

\subsection{The equations for linear elastodynamics}

We consider a three-dimensional elastic object represented by a bounded region $\Omega$ in $\mathbb{R}^{3}$ with smooth boundary $\partial \Omega$. We denote a point in $\Omega$ by $x=\left(x_{1}, x_{2}, x_{3}\right)$. The density and elastic parameters are modeled by the $C^{\infty}(\bar{\Omega})$ smooth function $\rho(x)$ and rank-4 tensor field $\mathbf{C}(x)$. The elasticity tensor in the case of general hyperelasticity has the symmetry properties

$$
\mathrm{C}_{i j k l}=\mathrm{C}_{k l i j}=\mathrm{C}_{j i k l}=\mathrm{C}_{i j l k}
$$

and is (uniformly) strongly elliptic on $\Omega$ if there is a constant $c>0$ such that, for any $x \in \Omega, \mathrm{C}_{i j k l}(x) V^{i} W^{j} V^{k} W^{l} \geq$ $c|\mathbf{V}|^{2}|\mathbf{W}|^{2}$ for all vectors $\mathbf{V}, \mathbf{W}$. The elasticity tensor is positive-definite on $\Omega$ if, for any $x \in \Omega, C_{i j k l}(x) E^{i j} E^{k l}>0$ for any symmetric matrix $E$.

The displacement after time $t$ of a point initially at position $x$ is represented by the vector-valued function $\mathbf{u}(x, t)=\left(u_{1}(x, t), u_{2}(x, t), u_{3}(x, t)\right)$ associated with the system of differential operators (1). Strong ellipticity is

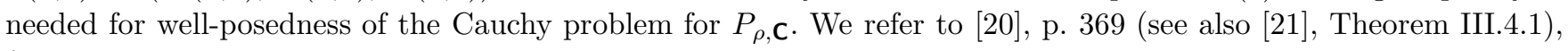
for conditions under which existence and uniqueness holds. 


\subsection{Spectral decomposition of the elasticity tensor for transversely isotropic elastodynamics}

Our aim in this paper is to study transversely isotropic elastodynamics from a basis and coordinate-invariant point of view. This approach is critical in the differential-geometric setting of [2], [17], for example. There, and in current work, we consider the pullback of an elasticity tensor, and study the type of anisotropy that occurs in the orbits of anisotropic elasticity tensors under the action of pullback by a diffeomorphism.

We begin by considering the spectral representation of the elasticity tensor $\mathbf{C}$ for transversely isotropic elastodynamics, obtained by Theocaris and Philippidis in $[16,(5),(12),(29),(30),(31)]$, as

$$
\begin{aligned}
C^{i j k l}=\quad \lambda_{1} & \cdot \frac{1}{2}\left[b^{i k} b^{j l}+b^{i l} b^{j k}-b^{i j} b^{k l}\right] \\
& +\lambda_{2} \cdot \frac{1}{2}\left[b^{i k} a^{j l}+b^{i l} a^{j k}+a^{i k} b^{j l}+a^{i l} b^{j k}\right]+\lambda_{3}\left[f^{i j} f^{k l}\right]+\lambda_{4}\left[g^{i j} g^{k l}\right],
\end{aligned}
$$

where the matrices $\boldsymbol{a}, \boldsymbol{b}, \boldsymbol{f}$, and $\boldsymbol{g}$ are given in terms of $\mathbf{k}$, a unit vector in the direction of the axis of transverse isotropy, and $\omega$, a scalar defined in terms of material parameters in (9), by

$$
\begin{aligned}
& \boldsymbol{a}=\mathbf{k} \otimes \mathbf{k}, \quad \boldsymbol{b}=\mathbf{I}-\boldsymbol{a}, \\
& \boldsymbol{f}=\boldsymbol{a} \sin (\omega)+\boldsymbol{b} \cos (\omega) / \sqrt{2}, \quad \boldsymbol{g}=-\boldsymbol{a} \cos (\omega)+\boldsymbol{b} \sin (\omega) / \sqrt{2} \text {. }
\end{aligned}
$$

In vector form

$$
\mathbf{C}=\frac{\lambda_{1}}{2}[\boldsymbol{b} \otimes \boldsymbol{b}-\boldsymbol{b} \otimes \boldsymbol{b}]+\lambda_{2}[\boldsymbol{b} \otimes \boldsymbol{a}]+\lambda_{3}[\boldsymbol{f} \otimes \boldsymbol{f}]+\lambda_{4}[\boldsymbol{g} \otimes \boldsymbol{g}]
$$

where $\otimes$ is the usual tensor product $(\boldsymbol{A} \otimes \boldsymbol{B})^{i j k l}=A^{i j} B^{k l}$, and $\otimes$, and $\Leftrightarrow$ are defined by

$$
(\boldsymbol{A} \otimes \boldsymbol{B})^{i j k l}=A^{i k} B^{j l}+A^{i l} B^{j k}, \quad \boldsymbol{A} \Leftrightarrow \boldsymbol{B}=\frac{1}{2}(\boldsymbol{A} \otimes \boldsymbol{B}+\boldsymbol{B} \otimes \boldsymbol{A}) .
$$

The elasticity tensor $\mathbf{C}$ is positive-definite iff the eigenvalues $\lambda_{i}$ are all positive.

By $[16,(28),(31)]$ we write the spectral representation in terms of the material parameters (the transverse and longitudinal Young's moduli $E_{T}, E_{L}$, Poisson's ratios $\nu_{T}, \nu_{L}$, and shear moduli $\left.\mu_{T}, \mu_{L}\right)$ :

$$
\begin{gathered}
\lambda_{1}=2 \mu_{T}, \quad \lambda_{2}=2 \mu_{L}, \quad \lambda_{3}=\frac{1}{2}(s+d), \quad \lambda_{4}=\frac{1}{2}(s-d), \\
s=\frac{r^{2}(m+t)}{2 r^{2} m t-1}, \quad d=-\frac{|r| \sqrt{r^{2}(m-t)^{2}+2}}{2 r^{2} m t-1} \quad \text { or } \quad d=0, \\
\cos (2 \omega)=\frac{|r|(m-t)}{\sqrt{r^{2}(m-t)^{2}+2}}, \quad m=\frac{1-\nu_{T}}{2 E_{T}}, \quad t=\frac{1}{2 E_{L}}, \quad r=\frac{E_{L}}{\nu_{L}} .
\end{gathered}
$$

We introduce the notation $\sigma_{\sin }= \pm 1$, where $\sin (2 \omega)=-\sigma_{\sin } \sqrt{2} / \sqrt{r^{2}(m-t)^{2}+2}$, and the sign $\sigma_{\text {sin }}$ is determined by $\sigma_{\text {sin }}=C /|C|=\mathbf{C}\left[\mathbf{k}, \mathbf{k}, \mathbf{k}^{\perp}, \mathbf{k}^{\perp}\right] /\left|\mathbf{C}\left[\mathbf{k}, \mathbf{k}, \mathbf{k}^{\perp}, \mathbf{k}^{\perp}\right]\right|$. Here, $\mathbf{k}^{\perp}$ represents any vector orthogonal to $\mathbf{k}$. (See (10) and (14).) We also recall the relation $2 \mu_{T}=E_{T} /\left(1+\nu_{T}\right)$.

\section{The slowness surface for general transversely isotropic elastic media}

We simplify the coordinate-invariant spectral representation (9) of the transversely isotropic elasticity tensor

(7) by introducing the following combinations $A, B, C$ of the material parameters (the transverse and longitudinal Young's moduli $E_{T}, E_{L}$, Poisson's ratios $\nu_{T}, \nu_{L}$, and shear moduli $\left.\mu_{T}, \mu_{L}\right)$. 
Definition of certain combinations of the material parameters. Let

$$
A=\frac{E_{L} E_{T}}{2\left[E_{L}\left(1-\nu_{T}\right)-2 E_{T} \nu_{L}^{2}\right]}, \quad B=\frac{E_{L}^{2}\left(1-\nu_{T}\right)}{E_{L}\left(1-\nu_{T}\right)-2 E_{T} \nu_{L}^{2}}, \quad C=\frac{\sigma_{s i n} E_{L}\left|\nu_{L}\right| E_{T}}{E_{L}\left(1-\nu_{T}\right)-2 E_{T} \nu_{L}^{2}}
$$

In terms of the spectral representation of $\mathbf{C}$ these parameters are $A=[s+d \cos (2 \omega)] / 4, \quad B=[s-d \cos (2 \omega)] / 2, \quad C=$ $\sqrt{2} d \sin (2 \omega) / 4$. We observe in the isotropy example below that $A+\mu_{T}, B$, and $C+2 \mu_{T}$ are generalizations of the expression $\lambda+2 \mu$ for isotropic elastic media.

We relate $A, B, C, \mu_{T}, \mu_{L}$ to the components $c_{i j}$ (with respect to an orthonormal basis $\left\{e_{1}, e_{2}, e_{3}\right\}$ with $e_{3}=k$ ) of the transversely isotropic elasticity tensor $\mathbf{C}$ by comparing the presentation of the slowness surfaces in [12], [13], [14], with those we derive in Section 4:

$$
\mu_{T}=\left(c_{11}-c_{12}\right) / 2=c_{66}, \quad \mu_{L}=c_{44}, \quad A=\left(c_{11}+c_{12}\right) / 2, \quad B=c_{33}, \quad C=c_{13},
$$

where $c_{i j}=\mathbf{C}\left[e_{i}, e_{i}, e_{j}, e_{j}\right], i, j=1,2,3 ; c_{i j}=0, i \neq j=4,5,6 ; c_{11}=c_{22}, c_{13}=c_{23}, c_{44}=c_{55}=\mathbf{C}\left[e_{2}, e_{3}, e_{2}, e_{3}\right], c_{66}=$ $\mathbf{C}\left[e_{1}, e_{2}, e_{1}, e_{2}\right]$.

Writing the material parameters $\nu_{T}, \nu_{L}, \boldsymbol{E}_{T}, \boldsymbol{E}_{\boldsymbol{L}}$ in terms of $\boldsymbol{A}, \boldsymbol{B}, \boldsymbol{C}, \boldsymbol{\mu}_{\boldsymbol{T}}$. Given (9) and (10), we write

$$
\begin{gathered}
E_{L}=\frac{1}{2 t}=\frac{A B-C^{2}}{2 A}, \quad E_{T}=\frac{4 \mu_{T}}{1+4 \mu_{T} m}=\frac{4 \mu_{T}\left(A B-C^{2}\right)}{A B-C^{2}+2 \mu_{T} B}, \\
\nu_{L}=\frac{1}{2 r t}= \pm \frac{\sqrt{A B+C^{2}}}{2 \sqrt{2} A}, \\
s=2 A+B, \quad d \cos (2 \omega)=2 A-B, \quad \sqrt{2} d \sin (2 \omega)=4 C, \quad \nu^{2}=(2 A-B)^{2}+8 C^{2} .
\end{gathered}
$$

(Compare with $[11,(2.13),(2.18),(2.19),(2.20)]$.

Positive-definiteness conditions. The elasticity tensor $\mathbf{C}$ is positive-definite at a material point $x$ if $C_{i j k l}(x) E^{i j} E^{k l}$ $>0$ for any symmetric matrix $E$. In the case of transverse isotropy the elasticity tensor $\mathbf{C}$ is positive-definite if and only if $\lambda_{i}>0, i=1, \ldots, 4$, which is equivalent to

since

$$
\mu_{T}>0, \quad \mu_{L}>0, \quad A>0, \quad B>0, \quad A B>C^{2}
$$

$$
\begin{gathered}
\lambda_{1}=2 \mu_{T}=c_{11}-c_{12}>0, \quad \lambda_{2}=2 \mu_{L}=c_{44}>0, \quad 2 A=c_{11}+c_{12}>0, \\
\quad \text { and } \quad \lambda_{3} \lambda_{4}=\frac{s^{2}-d^{2}}{4}=2\left(A B-C^{2}\right)=\left(c_{11}+c_{12}\right) c_{33}-2 c_{13}^{2}>0 .
\end{gathered}
$$

In fact, it follows from $A>0$ and $A B>C^{2}>0$ that $B>0$ and $\lambda_{3} \lambda_{4}>0$, and so $\lambda_{3}+\lambda_{4}=s=2 A+B>0$. Therefore, $\lambda_{3}, \lambda_{4}>0$. On the other hand, if $\lambda_{3}, \lambda_{4}>0$, then $A B>C^{2}>0$ and $2 A+B=s=\lambda_{3}+\lambda_{4}>0$, and so $A>0$ and $B=c_{33}>0$.

Strong ellipticity conditions. The elasticity tensor $\mathbf{C}$ is strongly elliptic at a material point $x$ if $C_{i j k l}(x) V^{i} W^{j} V^{k} W^{l}$ $>0$ for all vectors $\mathbf{V}, \mathbf{W}$. The strong ellipticity of $\mathbf{C}$ is equivalent to the positive-definiteness of the associated acoustical tensor $\mathbf{Q}(\mathbf{n})$. Necessary and sufficient conditions for strong ellipticity of the transversely isotropic elasticity tensor are given by Merodio and Ogden $[22,(2.9),(2.12)]$ to be

$$
c_{11}>0, \quad c_{33}>0, \quad c_{44}>0, \quad c_{11}>c_{12}, \quad\left|c_{13}+c_{44}\right|<c_{44}+\sqrt{c_{11} c_{33}}
$$

in terms of components $c_{i j}$ of the elasticity tensor with respect to an orthonormal basis $\left\{e_{1}, e_{2}, e_{3}\right\}$ with $e_{3}=k$. That is, $\mathbf{C}$ is strongly elliptic if and only if

$$
\begin{gathered}
\mu_{T}>0, \quad \mu_{L}>0, \quad A+\mu_{T}>0, \quad B>0, \quad \text { and } \\
\left|\mu_{L}+C\right|<\mu_{L}+\sqrt{\left(A+\mu_{T}\right) B} .
\end{gathered}
$$


Computing the principal symbol of the operator for transversely isotropic elastodynamics. Propagation of singularities for (1) is described in terms of the behaviour of the matrix-valued principal symbol $-\rho \tau^{2} \mathbf{I}+\mathbf{C}[\cdot, \boldsymbol{\xi}, \cdot, \boldsymbol{\xi}]$ of the operator $P_{\rho, \mathbf{C}}$ for elastodynamics. The principal symbol of the elliptic part agrees with the acoustical tensor $\mathbf{Q}(\mathbf{n})$ from the elasticity literature (e.g., [23]) when $|\boldsymbol{\xi}|=1$. The so-called wave normal $\mathbf{n}$ corresponds to $\boldsymbol{\xi} /|\boldsymbol{\xi}|$, where $\boldsymbol{\xi}$ is the dual vector to $x$.

We first write the transversely isotropic elasticity tensor $\mathbf{C}$ in an especially simple form. We define $S$ for vectors $\mathbf{v}, \mathbf{w}$ by $(\mathbf{v} S \mathbf{w})^{i j}=(1 / 2)\left(v^{i} w^{j}+w^{i} v^{j}\right)$ and for 2 -tensors $\boldsymbol{A}, \boldsymbol{B}$ by

$$
(\boldsymbol{A} S \boldsymbol{B})^{i j k l}=\frac{1}{2}(\boldsymbol{A} \otimes \boldsymbol{B}+\boldsymbol{B} \otimes \boldsymbol{A})^{i j k l}=\frac{1}{2}\left(A^{i j} B^{k l}+B^{i j} A^{k l}\right) .
$$

Then, referring to the spectral representation (7), (9), we write $\mathbf{C}$ explicitly only in terms of scalars, the identity matrix $\mathbf{I}$, and the fiber direction $\mathbf{k}$ (via $\mathbf{a}=\mathbf{k} \otimes \mathbf{k}$ ). (See [11, (2.2)], for example, for a presentation in terms of components $c_{i j}$ of the elasticity tensor given in terms of special bases.) In particular, we conclude that the elasticity tensor for transversely isotropic elastodynamics is given by

$$
\begin{aligned}
\mathbf{C}=(\mathbf{I} \otimes \mathbf{I}) \mu_{T}+2(\mathbf{I} \diamond \mathbf{a})\left(\mu_{L}-\mu_{T}\right)+(\mathbf{I} \otimes \mathbf{I})\left(A-\mu_{T}\right) \\
+(\mathbf{a} \otimes \mathbf{a})\left(A+\mu_{T}+B-2 C-4 \mu_{L}\right)+2(\mathbf{I} S \mathbf{a})\left(-A+\mu_{T}+C\right) .
\end{aligned}
$$

Given (14), we conclude that the principal symbol of the operator $P_{\rho, \mathbf{C}}$ for transversely isotropic elastodynamics has the form $-\rho \tau^{2} \mathbf{I}+\mathbf{C}[\cdot, \boldsymbol{\xi}, \cdot, \boldsymbol{\xi}]$, where

$$
\begin{gathered}
\mathbf{C}[\cdot, \boldsymbol{\xi}, \cdot, \boldsymbol{\xi}]=\alpha \mathbf{I}+\beta(\mathbf{k} \otimes \mathbf{k})+2 \gamma(\mathbf{k} S \boldsymbol{\xi})+\delta(\boldsymbol{\xi} \otimes \boldsymbol{\xi}), \\
\alpha=\alpha_{0}|\boldsymbol{\xi}|^{2}+\alpha_{1}(\mathbf{k} \cdot \boldsymbol{\xi})^{2}, \quad \delta=2 \delta_{0}, \quad \beta=2 \beta_{0}|\boldsymbol{\xi}|^{2}+2 \beta_{1}(\mathbf{k} \cdot \boldsymbol{\xi})^{2}, \\
\gamma(\mathbf{k} \cdot \boldsymbol{\xi})=\gamma_{1}(\mathbf{k} \cdot \boldsymbol{\xi})^{2}, \quad \alpha_{0}=\mu_{T}, \quad \alpha_{1}=2 \beta_{0}=\mu_{L}-\mu_{T}, \\
2 \delta_{0}=A, \quad 2 \beta_{1}=A+\mu_{T}+B-2 C-4 \mu_{L}, \quad \gamma_{1}=-A+C+\mu_{L} .
\end{gathered}
$$

Example: Isotropy. The transversely isotropic elasticity tensor reduces to the isotropic elasticity tensor $\mathbf{C}_{i s o}=$ $\lambda(\mathbf{I} \otimes \mathbf{I})+\mu(\mathbf{I} \otimes \mathbf{I})$, where $\lambda$ and $\mu$ are the Lamé parameters, if and only if

$$
\mu_{T}=\mu_{L}=\mu, \quad A=\lambda+\mu, \quad B=\lambda+2 \mu, \quad C=\lambda .
$$

The spectral representation of the isotropic elasticity tensor is $\mathbf{C}_{i s o}=(3 \lambda+2 \mu)(\mathbf{I} \otimes \mathbf{I}) / 3+\mu[\mathbf{I} \otimes \mathbf{I}-2(\mathbf{I} \otimes \mathbf{I}) / 3]$. The positive-definiteness conditions (11) for isotropic elasticity are $3 \lambda+2 \mu>0$ and $\mu>0$ (which implies $\lambda+\mu>0$ ). The strong ellipticity conditions (12) and (13) hold if and only if $\mu>0$ and $\lambda+2 \mu>0$.

The principal symbol $-\rho \tau^{2} \mathbf{I}+\mathbf{C}[\cdot, \boldsymbol{\xi}, \cdot, \boldsymbol{\xi}]$ in this case is given by $\left(-\rho \tau^{2}+\mu|\boldsymbol{\xi}|^{2}\right) \mathbf{I}+(\lambda+\mu)(\boldsymbol{\xi} \otimes \boldsymbol{\xi})$, and so $\alpha_{0}=c_{s}^{2}, \alpha_{1}=0, \beta_{0}=0, \beta_{1}=0, \gamma_{1}=0, \delta_{0}=\left(c_{p}^{2}-c_{s}^{2}\right) / 2$.

Computing the eigenvalues of the principal symbol. We show in Section 7 that the determinant of the principal symbol of $P_{\rho, \mathrm{C}}$ factors as

where $\quad-\rho^{3}\left(\tau^{2}-c_{0}^{2}\right)\left(\tau^{2}-c_{-}^{2}\right)\left(\tau^{2}-c_{+}^{2}\right)$,

$$
\begin{aligned}
\rho c_{0}^{2}(\boldsymbol{\xi}) & =\mu_{T}\left|\boldsymbol{\xi}_{k^{\perp}}\right|^{2}+\mu_{L}\left|\boldsymbol{\xi}_{k}\right|^{2}, \\
2 \rho c_{ \pm}^{2}(\boldsymbol{\xi}) & =\left(A+\mu_{T}+\mu_{L}\right)\left|\boldsymbol{\xi}_{k^{\perp}}\right|^{2}+\left(B+\mu_{L}\right)\left|\boldsymbol{\xi}_{k}\right|^{2} \pm|D| \\
D^{2} & =\left(A+\mu_{T}-\mu_{L}\right)^{2}\left|\boldsymbol{\xi}_{k^{\perp}}\right|^{4}+\left(B-\mu_{L}\right)^{2}\left|\boldsymbol{\xi}_{k}\right|^{4}-2\left[\left(A+\mu_{T}-\mu_{L}\right)\left(B-\mu_{L}\right)-2\left(C+\mu_{L}\right)^{2}\right]\left|\boldsymbol{\xi}_{k^{\perp}}\right|^{2}\left|\boldsymbol{\xi}_{k}\right|^{2} .
\end{aligned}
$$

Here we decompose $\boldsymbol{\xi}=\boldsymbol{\xi}_{k^{\perp}}+\boldsymbol{\xi}_{k}$, with $\boldsymbol{\xi}_{k^{\perp}}=\boldsymbol{\xi}-(\boldsymbol{\xi} \cdot \mathbf{k}) \mathbf{k}$ the component of $\boldsymbol{\xi}$ orthogonal to $\mathbf{k}$, and $\boldsymbol{\xi}_{k}=(\boldsymbol{\xi} \cdot \mathbf{k}) \mathbf{k}$ the component of $\boldsymbol{\xi}$ in the direction of $\mathbf{k}$. 
The wave speed $\boldsymbol{c}_{\boldsymbol{m}}(\boldsymbol{x}, \boldsymbol{\xi} /|\boldsymbol{\xi}|)$ in the direction $\boldsymbol{\xi}$. The terms $c_{m}^{2}(x, \boldsymbol{\xi})$ in the determinant of the principal symbol of $P_{\rho, \mathbf{C}}$ are the eigenvalues of its elliptic part, and correspond to the wave speeds $c_{m}(x, \boldsymbol{\xi} /|\boldsymbol{\xi}|)$ of the wave modes associated with the wave normal direction $\boldsymbol{\xi} /|\boldsymbol{\xi}|$. (For isotropic elastodynamics the wave speeds are $c(x, \boldsymbol{\xi} /|\boldsymbol{\xi}|)=c_{p}(x), c_{s}(x)$.) In the case that $\mu_{L}=A+\mu_{T}=B$, for example, the wave speeds are ordered by $c_{-} \leq c_{0} \leq c_{+}$, where $c_{+}$is the quasi-compressional wave speed, $c_{-}$is the quasi-shear wave speed, and $c_{0}$ is the pure shear wave speed. On the other hand, if $A<0$ (e.g. when $\mathbf{C}$ is strongly elliptic, but not positive-definite), if $B<\mu_{L}$, and if $C+\mu_{L}$ is small enough, then the wave speeds are ordered by $c_{-} \leq c_{+} \leq c_{0}$.

The sheets $1=c_{m}^{2}(x, s)$ of the slowness surface. The slowness surface consists of the points $(x, \mathrm{~s})$ that satisfy the characteristic condition (2). The slowness surface for a fixed $x$ is rotationally invariant with respect to $\mathbf{s}$ in the plane orthogonal to $\mathbf{k}(x)$; this plane is called the isotropic plane, or the basal plane. The sheets $1=c_{m}^{2}(x, s)$ of the slowness surface are given via $\mathbf{s}=\boldsymbol{\xi} / \tau$ in terms of the eigenvalues $c_{m}^{2}(x, \boldsymbol{\xi})$ of the elliptic part of the principal symbol of $P_{\rho, \mathbf{C}} / \rho$.

\section{Conditions for ellipsoidal transversely isotropic elastic media}

Pointwise conditions so that the slowness surface is the union of ellipsoids. The slowness surface is the union of ellipsoids if the factors $\tau^{2}-c^{2}(x, \boldsymbol{\xi})$ have $c^{2}=p \pm|q|$ with $p(x, \boldsymbol{\xi}), q(x, \boldsymbol{\xi})$ that are homogeneous polynomials of degree 2 in $\boldsymbol{\xi}$. It follows from factoring the determinant of the principal symbol of $P_{\rho, \mathbf{C}}$ that the slowness surface is the union of ellipsoids if and only if one of the two following conditions holds:

(Q1) $\mu_{L}+C=0, \quad$ i.e., $c_{13}+c_{44}=0 ;$

(Q2) $\left(\mu_{L}+C\right)^{2}=\left(A+\mu_{T}-\mu_{L}\right)\left(B-\mu_{L}\right)$, which is equivalent to each of:

$$
\left(c_{13}+c_{44}\right)^{2}=\left(c_{11}-c_{44}\right)\left(c_{33}-c_{44}\right), \quad \mu_{L}=\frac{\left(A+\mu_{T}\right) B-C^{2}}{A+\mu_{T}+B+2 C} .
$$

See $[13$, p. $656,(3.13),(6.13)]$, and $\left[12,(1.1),(1.2),(C 3)_{1},(P 3)_{1}\right]$ for a derivation of these conditions in terms of the components $c_{i j}$ of the elasticity tensor with respect to a special basis. The notation used in these articles is similar to that used here: $a_{1}=\left(A+\mu_{T}\right) / \rho, a_{2}=\mu_{T} / \rho, a_{3}=B / \rho, a_{4}=\mu_{L} / \rho, a_{5}=\left(C+\mu_{L}\right) / \rho$. For example, in this notation $(Q 1)$ is given by $a_{5}=0$.

In these cases we can label the $c_{m}, m=1,2,3$, as $c_{0}, c_{ \pm}$, where

$$
\begin{aligned}
\rho c_{0}^{2}(\boldsymbol{\xi}) & =\mu_{T}\left|\boldsymbol{\xi}_{k^{\perp}}\right|^{2}+\mu_{L}\left|\boldsymbol{\xi}_{k}\right|^{2}, \\
\rho c_{ \pm}^{2}(\boldsymbol{\xi}) & =\left(A+\mu_{T}+\mu_{L}\right)\left|\boldsymbol{\xi}_{k^{\perp}}\right|^{2} / 2+\left(B+\mu_{L}\right)\left|\boldsymbol{\xi}_{k}\right|^{2} / 2 \pm\left.\left|\left(A+\mu_{T}-\mu_{L}\right)\right| \boldsymbol{\xi}_{k^{\perp}}\right|^{2} / 2+\sigma_{Q}\left(B-\mu_{L}\right)\left|\boldsymbol{\xi}_{k}\right|^{2} / 2 \mid,
\end{aligned}
$$

and $\sigma_{Q}=-1$ for $(Q 1)$, while $\sigma_{Q}=+1$ for $(Q 2)$.

Note that the absolute value is not needed in (19) in case (Q2), since $\left(C+\mu_{L}\right)^{2}=\left(A+\mu_{T}-\mu_{L}\right)\left(B-\mu_{L}\right)$ implies that one of $A+\mu_{T}-\mu_{L}$ and $B-\mu_{L}$ is zero, or $A+\mu_{T}-\mu_{L}$ and $B-\mu_{L}$ have the same sign (i.e., $\mu_{L}$ is smaller than, or larger than, both of $A+\mu_{T}$ and $B$ ).

The reduction of $(Q 1)$ to isotropy via (16) results in media that is strongly elliptic, but not positive-definite since $A=C+\mu=0$. The reduction of (Q2) to isotropy via (16) results in media that is both strongly elliptic and positive-definite.

In each of the cases $(Q 1)$ and (Q2) the strong ellipticity conditions (12) and (13) reduce to (12). In fact, the condition $\mu_{L}=\left[\left(A+\mu_{T}\right) B-C^{2}\right] /\left[A+\mu_{T}+B+2 C\right]$ for (Q2) may be written as $\mu_{L}\left(A+\mu_{T}+B\right)+\left(\mu_{L}+C\right)^{2}=$ $\left(A+\mu_{T}\right) B+\mu_{L}^{2}$, and so in the case of (Q2) (13) may be stated as $\mu_{L}\left(A+\mu_{T}+B\right)+\mu_{L}^{2}+2 \mu_{L} \sqrt{\left(A+\mu_{T}\right) B}+\left(A+\mu_{T}\right) B>$ $\left(A+\mu_{T}\right) B+\mu_{L}^{2}$, which holds trivially given (12).

Pointwise conditions so that each of the sheets $1=c_{m}^{2}(x, s)$ of the slowness surface is an ellipsoid. Under additional conditions each of the $c_{m}^{2}(x, \boldsymbol{\xi})$ in case $(Q 1)$ is a homogeneous polynomial of degree 2 in $\boldsymbol{\xi}$ (in particular, $c_{m}^{2}(x, \boldsymbol{\xi})$ is a smooth function of position $x$ and direction $\left.\boldsymbol{\xi}\right)$, and each of the sheets $1=c_{m}^{2}(x, s)$ of the 
corresponding slowness surface is ellipsoidal. (Q2) already has these properties. It follows that for (Q2), and for (Q1) under the additional conditions, wave propagation through the interior for each wave mode (see [2]) occurs along geodesic segments of the Riemannian metric given by $G_{m}^{i j}(x) \xi_{i} \xi_{j}=c_{m}^{2}(x, \boldsymbol{\xi})$. We refer to wave propagation along geodesic segments as geodesic wave propagation (GWP).

We show in Section 7 that each term $c_{m}^{2}$ in the factors $\tau^{2}-c_{m}^{2}(x, \boldsymbol{\xi})$ of the determinant of the principal symbol of $P_{\rho, \mathrm{C}}$ is a polynomial in $\boldsymbol{\xi}$ if and only if one of the two following conditions holds:

$$
\begin{aligned}
& \mu_{L}+C=0, \text { and } B \leq \mu_{L} \leq A+\mu_{T} \quad \text { or } \quad A+\mu_{T} \leq \mu_{L} \leq B \\
& \left(\mu_{L}+C\right)^{2}=\left(A+\mu_{T}-\mu_{L}\right)\left(B-\mu_{L}\right) .
\end{aligned}
$$

In these cases we can label the $c_{m}, m=1,2,3$, as $c_{0}, c_{ \pm}$, where $\rho c_{0}^{2}(\boldsymbol{\xi})=\mu_{T}\left|\boldsymbol{\xi}_{k^{\perp}}\right|^{2}+\mu_{L}\left|\boldsymbol{\xi}_{k}\right|^{2}$, and

for $(G W P 1)$

$$
\begin{array}{lll}
\text { if } \quad B \leq \mu_{L} \leq A+\mu_{T}, & \text { then } \rho c_{+}^{2}=\left(A+\mu_{T}\right)\left|\boldsymbol{\xi}_{k^{\perp}}\right|^{2}+\mu_{L}\left|\boldsymbol{\xi}_{k}\right|^{2}, & \rho c_{-}^{2}=\mu_{L}\left|\boldsymbol{\xi}_{k^{\perp}}\right|^{2}+B\left|\boldsymbol{\xi}_{k}\right|^{2}, \\
\text { if } \quad A+\mu_{T} \leq \mu_{L} \leq B, & \text { then } \rho c_{+}^{2}=\mu_{L}\left|\boldsymbol{\xi}_{k^{\perp}}\right|^{2}+B\left|\boldsymbol{\xi}_{k}\right|^{2}, & \rho c_{-}^{2}=\left(A+\mu_{T}\right)\left|\boldsymbol{\xi}_{k^{\perp}}\right|^{2}+\mu_{L}\left|\boldsymbol{\xi}_{k}\right|^{2} ;
\end{array}
$$

for $(G W P 2)$

$$
\begin{array}{lll}
\text { if } \mu_{L} \leq A+\mu_{T}, \mu_{L} \leq B, \quad \text { then } \rho c_{+}^{2}=\left(A+\mu_{T}\right)\left|\boldsymbol{\xi}_{k^{\perp}}\right|^{2}+B\left|\boldsymbol{\xi}_{k}\right|^{2}, & \rho c_{-}^{2}=\mu_{L}\left|\boldsymbol{\xi}_{k^{\perp}}\right|^{2}+\mu_{L}\left|\boldsymbol{\xi}_{k}\right|^{2}, \\
\text { if } \quad A+\mu_{T} \leq \mu_{L}, B \leq \mu_{L}, & \text { then } \rho c_{+}^{2}=\mu_{L}\left|\boldsymbol{\xi}_{k^{\perp}}\right|^{2}+\mu_{L}\left|\boldsymbol{\xi}_{k}\right|^{2}, & \rho c_{-}^{2}=\left(A+\mu_{T}\right)\left|\boldsymbol{\xi}_{k^{\perp}}\right|^{2}+B\left|\boldsymbol{\xi}_{k}\right|^{2} .
\end{array}
$$

In other words, in case (GWP1) the sheets of the slowness surface are given by:

$$
1=\widehat{\mu}_{T}\left|\mathbf{s}_{k^{\perp}}\right|^{2}+\widehat{\mu}_{L}\left|\mathbf{s}_{k}\right|^{2}, \quad 1=\left(\widehat{A}+\widehat{\mu}_{T}\right)\left|\mathbf{s}_{k^{\perp}}\right|^{2}+\widehat{\mu}_{L}\left|\mathbf{s}_{k}\right|^{2}, \quad 1=\widehat{\mu}_{L}\left|\mathbf{s}_{k^{\perp}}\right|^{2}+\widehat{B}\left|\mathbf{s}_{k}\right|^{2}
$$

while in case (GWP2) they are:

$$
1=\widehat{\mu}_{T}\left|\mathbf{s}_{k^{\perp}}\right|^{2}+\widehat{\mu}_{L}\left|\mathbf{s}_{k}\right|^{2}, \quad 1=\widehat{\mu}_{L}|\mathbf{s}|^{2}, \quad 1=\left(\widehat{A}+\widehat{\mu}_{T}\right)\left|\mathbf{s}_{k^{\perp}}\right|^{2}+\widehat{B}\left|\mathbf{s}_{k}\right|^{2},
$$

where $\mathbf{s}_{k^{\perp}}=s-(s \cdot k) k, \quad \mathbf{s}_{k}=(s \cdot k) k, \quad \widehat{\mu}_{T}=\mu_{T} / \rho, \quad \widehat{\mu}_{L}=\mu_{L} / \rho, \quad \widehat{A}=A / \rho, \quad \widehat{B}=B / \rho, \quad \widehat{C}=C / \rho$.

Examples: Ellipsoidal cases close to isotropy. An example of transverse isotropy with GWP is the case with both (GWP1) and (GWP2):

$$
\mu_{L}=A+\mu_{T}=B=-C, \text { i.e., } c_{11}=c_{33}=c_{44}=-c_{13} .
$$

The sheets of the slowness surface in this case are:

$$
1=\widehat{\mu}_{T}\left|\mathbf{s}_{k^{\perp}}\right|^{2}+\widehat{\mu}_{L}\left|\mathbf{s}_{k}\right|^{2}, \quad 1=\widehat{\mu}_{L}|\mathbf{s}|^{2}, \quad 1=\widehat{\mu}_{L}|\mathbf{s}|^{2} .
$$

This case reduces to isotropic iff $\mu_{T}=\mu_{L}$ (iff $A=0$ ).

Another example is that of (GWP2) with $\mu_{T}=\mu_{L}$ :

$$
\begin{aligned}
(G \text { WP2iso }) & \left(C+\mu_{L}\right)^{2}=\left(A+\mu_{T}-\mu_{L}\right)\left(B-\mu_{L}\right) \text { and } \mu_{T}=\mu_{L}, \\
\text { i.e., } & 2 \mu_{T}=2 \mu_{L}=-(A+2 C)+\sqrt{A^{2}+4 A(B+C)}, \\
\text { i.e., } \quad & 4 c_{44}=4 c_{66}=-\left(c_{11}+5 c_{12}\right)+\sqrt{\left(c_{11}+c_{12}\right)\left(c_{11}+9 c_{12}+8 c_{33}\right)} .
\end{aligned}
$$

This expression for $\mu_{T}$ is real and positive since $A^{2}+4 A(B+C)>(A+2 C)^{2} \geq 0$ by (11).

The sheets of the slowness surface in this case are:

$$
1=\widehat{\mu}|\mathbf{s}|^{2}, \quad 1=\widehat{\mu}|\mathbf{s}|^{2}, \quad 1=(\widehat{A}+\widehat{\mu})\left|\mathbf{s}_{k^{\perp}}\right|^{2}+\widehat{B}\left|\mathbf{s}_{k}\right|^{2}
$$


Here $\mu=\mu_{T}=\mu_{L}$ and $\widehat{\mu}=\mu / \rho$. This case reduces to isotropic if and only if $A+\mu_{T}=B$. It follows that two eigenvalues may coincide for all directions of $\boldsymbol{\xi}$, without anisotropy degenerating to isotropy. (See also Chadwick's [11, p. 39, 44-45] description of this non-isotropic case (GWP2iso) of transverse isotropy with coincident shear modes.)

\section{Describing the type of intersection of the sheets of the slowness surface for transversely isotropic elastic media}

We see from the representation (18) of the quasi-compressional (QC), quasi-shear (QS), and pure shear (PS) wave speeds

$$
\begin{aligned}
2 \rho c_{Q C}^{2}(\boldsymbol{\xi}) & =\left(A+\mu_{T}+\mu_{L}\right)\left|\boldsymbol{\xi}_{k^{\perp}}\right|^{2}+\left(B+\mu_{L}\right)\left|\boldsymbol{\xi}_{k}\right|^{2}+|D|, \\
2 \rho c_{Q S}^{2}(\boldsymbol{\xi}) & =\left(A+\mu_{T}+\mu_{L}\right)\left|\boldsymbol{\xi}_{k^{\perp}}\right|^{2}+\left(B+\mu_{L}\right)\left|\boldsymbol{\xi}_{k}\right|^{2}-|D|, \\
\rho c_{P S}^{2}(\boldsymbol{\xi}) & =\mu_{T}\left|\boldsymbol{\xi}_{k^{\perp}}\right|^{2}+\mu_{L}\left|\boldsymbol{\xi}_{k}\right|^{2},
\end{aligned}
$$

where

$$
D^{2}=\left[\left(A+\mu_{T}-\mu_{L}\right)\left|\boldsymbol{\xi}_{k^{\perp}}\right|^{2}-\left(B-\mu_{L}\right)\left|\boldsymbol{\xi}_{k}\right|^{2}\right]^{2}+4\left(C+\mu_{L}\right)^{2}\left|\boldsymbol{\xi}_{k^{\perp}}\right|^{2}\left|\boldsymbol{\xi}_{k}\right|^{2},
$$

that the wave speeds (and sheets $1=c^{2}(s)$ of the slowness surface for transversely isotropic elastic media depend on $C$ only via $\left(C+\mu_{L}\right)^{2}$. In fact, we can view the class of transversely isotropic elastic media as the union of families with each family parametrized by what we will call the anellipticity parameter $a_{C}=C+\mu_{L}$. The term anellipticity refers to the fact that by varying this single parameter, we change the slowness surface only away from the fiber and the isotropic plane, and make the slowness surface elliptic only at the particular values $a_{C}=0$ (when the slowness surface is type $(Q 1))$ and $a_{C}=\sqrt{\left(A+\mu_{T}-\mu_{L}\right)\left(B-\mu_{L}\right)}$ if $\left(A+\mu_{T}-\mu_{L}\right)\left(B-\mu_{L}\right) \geq 0$ (when the slowness surface is type $($ Q2)).

In the following proposition we describe conditions under which the sheets of the slowness surface intersect. (Compare with Payton [9].) As a consequence, following the proposition we specify how the type of intersection depends on the anellipticity parameter $a_{C}$, and we plot cases representative of each type of intersection in Figures 1,2 , and 3. (In general, the type of intersection may change only when $a_{C}$ passes from $a_{C}=0$ to $a_{C}>0$ or when $a_{C}$ passes through the critical value $a_{C}^{2}=A\left(B-\mu_{L}\right)$.) Chadwick includes the critical anellipticity condition $\left(C+\mu_{L}\right)^{2}=A\left(B-\mu_{L}\right)$ in his classification of positive-definite, transversely isotropic elastic media in [11].

Proposition 1 (Conditions under which the sheets of the slowness surface intersect for positivedefinite, transversely isotropic elastic media) The quasi-compressional and pure shear sheets of the slowness surface intersect at the fiber if and only if $B \leq \mu_{L}$, and do not intersect otherwise.

The quasi-shear and pure shear sheets of the slowness surface intersect 1) at the fiber if and only if $B \geq \mu_{L}$, 2) on the cone $\left|\boldsymbol{\xi}_{k}\right|^{2} /\left|\boldsymbol{\xi}_{k^{\perp}}\right|^{2}=A\left(\mu_{T}-\mu_{L}\right) /\left[A\left(B-\mu_{L}\right)-\left(C+\mu_{L}\right)^{2}\right]$ if and only if $A\left(B-\mu_{L}\right)-\left(C+\mu_{L}\right)^{2} \neq 0$ and $\left.A\left(\mu_{T}-\mu_{L}\right) /\left[A\left(B-\mu_{L}\right)-\left(C+\mu_{L}\right)^{2}\right] \geq 0,3\right)$ everywhere if and only if $A\left(B-\mu_{L}\right)=\left(C+\mu_{L}\right)^{2}$ and $\mu_{T}=\mu_{L}$, and 4) nowhere otherwise.

The quasi-compressional and quasi-shear sheets of the slowness surface intersect 1) everywhere if and only if $A+\mu_{T}=\mu_{L}, B=\mu_{L}$, and $C+\mu_{L}=0$, 2) on the isotropic plane if and only if $A+\mu_{T}=\mu_{L}$, 3) at the fiber if and only if $B=\mu_{L}$, and 4) on the cone $\left|\boldsymbol{\xi}_{k}\right|^{2} /\left|\boldsymbol{\xi}_{k^{\perp}}\right|^{2}=\left(A+\mu_{T}-\mu_{L}\right) /\left(B-\mu_{L}\right)$ if and only if $\left(A+\mu_{T}-\mu_{L}\right) /\left(B-\mu_{L}\right) \geq 0, B \neq \mu_{L}$, and $C+\mu_{L}=0$, and 5) nowhere otherwise.

We prove Proposition 1 in Section 7.

We conclude from Proposition 1 that the type of intersection of the sheets of the slowness surface (in the case of positive-definiteness) depends on the anellipticity parameter $a_{C}$ in the following ways:

1) In the case that $A\left(B-\mu_{L}\right) \geq 0$ a) the quasi-shear and pure shear sheets of the slowness surface intersect at points away from the fiber and away from the isotropic plane when $A\left(\mu_{T}-\mu_{L}\right) /\left[A\left(B-\mu_{L}\right)-\left(C+\mu_{L}\right)^{2}\right]>0$, and these sheets do not intersect at the fiber or on the isotropic plane when, varying only $C, A\left(\mu_{T}-\mu_{L}\right) /\left[A\left(B-\mu_{L}\right)-\left(C+\mu_{L}\right)^{2}\right]$ becomes negative; b) in the case $\mu_{T}=\mu_{L}$ the quasi-shear and pure shear sheets are coincident at the critical value $\left(C+\mu_{L}\right)^{2}=A\left(B-\mu_{L}\right)$, while, if we vary $C$ so that $\left(C+\mu_{L}\right)^{2} \neq A\left(B-\mu_{L}\right)$, then they intersect only on the 
isotropic plane (and possibly at the fiber).

2) The quasi-compressional and quasi-shear sheets of the slowness surface are coincident if and only if $A+\mu_{T}=$ $\mu_{L}, \quad B=\mu_{L}$, and $C+\mu_{L}=0 ;$ when we vary only $C$ so that $C+\mu_{L} \neq 0$, the corresponding sheets of the slowness surface intersect only at the fiber and on the isotropic plane. In addition, these sheets intersect on the cone $\left|\boldsymbol{\xi}_{k}\right|^{2} /\left|\boldsymbol{\xi}_{k^{\perp}}\right|^{2}=\left(A+\mu_{T}-\mu_{L}\right) /\left(B-\mu_{L}\right)$ if and only if $\left(A+\mu_{T}-\mu_{L}\right) /\left(B-\mu_{L}\right) \geq 0, B \neq \mu_{L}, C+\mu_{L}=0$; but if $C$ is varied so that $C+\mu_{L} \neq 0$, then they intersect nowhere if $A+\mu_{T} \neq \mu_{L}$ and only on the isotropic plane if $A+\mu_{T}=\mu_{L}$.

Our results diverge slightly from those of Chadwick [11] and Chadwick and Shuvalov [23]. Chadwick's classification is based on the positivity or negativity of the combinations, $A+\mu_{T}-\mu_{L}=a_{1}-a_{4}, \mu_{T}-\mu_{L}=a_{2}-a_{4}, B-\mu_{L}=$ $a_{3}-a_{4}$, and $A\left(B-\mu_{L}\right)-\left(C+\mu_{L}\right)^{2}=$ Chadwick's $A$, of material parameters.

We observe that when $B<\mu_{L}$, we have that $A\left(B-\mu_{L}\right)$ is negative, and so there is no positive-definite, transversely isotropic elastic medium that is subcritical, that is, with $\left(C+\mu_{L}\right)^{2}<A\left(B-\mu_{L}\right)$, i.e., with Chadwick's "A", which is $A\left(B-\mu_{L}\right)-\left(C+\mu_{L}\right)^{2}$, positive. But the positive-definite, transversely isotropic elastic media with $B<\mu_{L}, \mu_{T}<A+\mu_{T}<\mu_{L}$, and $C+\mu_{L}=0$, have Chadwick classification $C_{33}$, and those with $B<\mu_{L}, \mu_{T}<$ $A+\mu_{T}=\mu_{L}$, and $C+\mu_{L}=0$ have Chadwick classification $D_{43}$, and are not included in the lists [11, (5.24)]. There it is required that $A\left(B-\mu_{L}\right)-\left(C+\mu_{L}\right)^{2}=$ Chadwick's $A=\left(a_{1}-a_{4}\right)+\left(a_{3}-a_{4}\right)>0$, which cannot hold when $B<\mu_{L}$ and $C+\mu_{L}=0$.

\section{More on ellipsoidal, strongly elliptic transverse isotropy}

In this section we first derive conditions under which strongly elliptic, ellipsoidal, transversely isotropic elastic media have a disjoint sheet of the slowness surface. We then describe the polarization vectors for transversely isotropic elastodynamics with $(G W P)$.

6.1 Conditions for a disjoint sheet of the slowness surface for ellipsoidal, strongly elliptic transversely isotropic elastodynamics with GWP

In Table 1 we summarize the cases of GWP for which the third mode is disjoint from the others. The vertical axis represents the fiber direction $\mathbf{k}$, and the horizontal axis represents any axis orthogonal to $\mathbf{k}$. (Slowness surfaces for transverse isotropy have a rotational symmetry around the fiber direction $\mathbf{k}$.) The circles in the figures have radius $1 / \sqrt{\mu_{L}}$, and the double circle represents two sheets of the slowness surface that coincide for all $\boldsymbol{\xi}$.

We emphasize that although the disjoint mode is the fastest in three of the four cases (since its wave speed $c(x, \boldsymbol{\xi})$ is larger than the others), in fact, the disjoint mode is the slowest in one case for (CM1), when $B<\mu_{L}<\mu_{T}<A+\mu_{T}$, i.e., $c_{33}<c_{44}<c_{66}<c_{11}$.

For convenience sake we introduce an arbitrary (but simpler) labeling of the wave speeds for $(G W P 1)$ as follows:

$$
\begin{gathered}
\rho \underline{c}_{0}^{2}=-\rho \tau^{2}+\mu_{T}\left|\boldsymbol{\xi}_{k^{\perp}}\right|^{2}+\mu_{L}\left|\boldsymbol{\xi}_{k}\right|^{2}, \quad \rho \underline{c}_{1}^{2}=-\rho \tau^{2}+\left(A+\mu_{T}\right)\left|\boldsymbol{\xi}_{k^{\perp}}\right|^{2}+\mu_{L}\left|\boldsymbol{\xi}_{k}\right|^{2}, \\
\rho \underline{c}_{2}^{2}=-\rho \tau^{2}+\mu_{L}\left|\boldsymbol{\xi}_{k^{\perp}}\right|^{2}+B\left|\boldsymbol{\xi}_{k}\right|^{2},
\end{gathered}
$$

and for (GWP2) by

$$
\begin{gathered}
\rho \underline{c}_{0}^{2}=-\rho \tau^{2}+\mu_{T}\left|\boldsymbol{\xi}_{k^{\perp}}\right|^{2}+\mu_{L}\left|\boldsymbol{\xi}_{k}\right|^{2}, \quad \rho \underline{c}_{1}^{2}=-\rho \tau^{2}+\mu_{L}\left|\boldsymbol{\xi}_{k^{\perp}}\right|^{2}+\mu_{L}\left|\boldsymbol{\xi}_{k}\right|^{2}, \\
\rho \underline{c}_{2}^{2}=-\rho \tau^{2}+\left(A+\mu_{T}\right)\left|\boldsymbol{\xi}_{k^{\perp}}\right|^{2}+B\left|\boldsymbol{\xi}_{k}\right|^{2} .
\end{gathered}
$$

The light cones are the locus of points $(t, x, \tau, \boldsymbol{\xi})$ with $\tau^{2}=c_{m}^{2}(x, \boldsymbol{\xi})$.

Corollary 2 (Conditions for a disjoint mode along rays for $(\boldsymbol{G W P 1})$ ) Rays in the first mode with $\boldsymbol{\xi}$ not parallel to $\mathbf{k}$ are bounded away from the light cones for the other modes under the following conditions on the material parameters: $\mu_{L}=B \neq \mu_{T}$, or $\mu_{L} \neq \mu_{T}$ and $\mu_{L}$ lies between $\mu_{T}$ and $B$. 
Fig. 1. Types of intersection of the sheets of the slowness surface for positive-definite, transversely isotropic elastic media in families that have distinct (Q1), (Q2), and critical anellipticity values. Recall that the anellipticity value $a_{C}=C+\mu_{L}$ for $(Q 1)$ is given by $a_{C}=0$, for $(Q 2)$ by $a_{C}^{2}=\left(A+\mu_{T}-\mu_{L}\right)\left(B-\mu_{L}\right)$, and in the critical case is given by $a_{C}^{2}=A\left(B-\mu_{L}\right)$. The sheets of the slowness surface are plotted slightly offset in the case $a_{C}=0$.

\begin{tabular}{|c|c|c|c|c|}
\hline \multirow{2}{*}{$\begin{array}{l}\text { Conditions } \\
\text { on } C+\mu_{L}\end{array}$} & $\begin{array}{c}\text { Conditions } \\
\text { on } A, B, \mu_{L}, \mu_{T}\end{array}$ & \multirow{2}{*}{$\begin{array}{l}\text { Conditions } \\
\text { on } C+\mu_{L}\end{array}$} & \multicolumn{2}{|c|}{ Conditions on $A, B, \mu_{L}, \mu_{T}$} \\
\hline & $\begin{array}{c}B>\mu_{L} \\
\mu_{L}<\mu_{T}<A+\mu_{T}\end{array}$ & & $\begin{array}{c}B>\mu_{L} \\
\mu_{L}=\mu_{T}<A+\mu_{T}\end{array}$ & $\begin{array}{c}B>\mu_{L} \\
\mu_{T}<\mu_{L}<A+\mu_{T}\end{array}$ \\
\hline$C+\mu_{L}=0$ & & $C+\mu_{L}=0$ & & \\
\hline $\begin{array}{c}0<\left(C+\mu_{L}\right)^{2} \\
<A\left(B-\mu_{L}\right)\end{array}$ & & $\begin{array}{c}0<\left(C+\mu_{L}\right)^{2} \\
<\left(A+\mu_{T}-\mu_{L}\right) \\
\quad \cdot\left(B-\mu_{L}\right)\end{array}$ & & \\
\hline $\begin{array}{c}\left(C+\mu_{L}\right)^{2}= \\
A\left(B-\mu_{L}\right)\end{array}$ & & $\begin{array}{c}\left(C+\mu_{L}\right)^{2}= \\
\left(A+\mu_{T}-\mu_{L}\right) \\
\quad \cdot\left(B-\mu_{L}\right)\end{array}$ & & \\
\hline $\begin{array}{l}A\left(B-\mu_{L}\right) \\
<\left(C+\mu_{L}\right)^{2} \\
<\left(A+\mu_{T}-\mu_{L}\right) \\
\quad \cdot\left(B-\mu_{L}\right)\end{array}$ & & $\begin{array}{c}\left(A+\mu_{T}-\mu_{L}\right) \\
\quad \cdot\left(B-\mu_{L}\right) \\
<\left(C+\mu_{L}\right)^{2} \\
<A\left(B-\mu_{L}\right)\end{array}$ & & \\
\hline $\begin{array}{c}\left(C+\mu_{L}\right)^{2}= \\
\left(A+\mu_{T}-\mu_{L}\right) \\
\quad \cdot\left(B-\mu_{L}\right)\end{array}$ & & $\begin{array}{c}\left(C+\mu_{L}\right)^{2}= \\
A\left(B-\mu_{L}\right)\end{array}$ & & \\
\hline $\begin{array}{c}\left(C+\mu_{L}\right)^{2}> \\
\left(A+\mu_{T}-\mu_{L}\right) \\
\cdot\left(B-\mu_{L}\right)\end{array}$ & & $\begin{array}{c}\left(C+\mu_{L}\right)^{2}> \\
A\left(B-\mu_{L}\right)\end{array}$ & & \\
\hline
\end{tabular}


Fig. 2. Types of intersection of the sheets of the slowness surface for positive-definite, transversely isotropic elastic media in families that have exactly two distinct $(Q 1)$, (Q2), and critical anellipticity values.

\begin{tabular}{|c|c|c|c|c|}
\hline \multirow{2}{*}{$\begin{array}{l}\text { Conditions } \\
\text { on } C+\mu_{L}\end{array}$} & \multicolumn{2}{|c|}{ Conditions on $A, B, \mu_{L}, \mu_{T}$} & \multirow{2}{*}{$\begin{array}{l}\text { Conditions } \\
\text { on } C+\mu_{L}\end{array}$} & Conditions \\
\hline & $\begin{array}{c}B>\mu_{L} \\
\mu_{T}<\mu_{L}=A+\mu_{T}\end{array}$ & $\begin{array}{c}B>\mu_{L} \\
\mu_{T}<A+\mu_{T}<\mu_{L}\end{array}$ & & $\begin{array}{c}B<\mu_{L} \\
\mu_{T}<A+\mu_{T}<\mu_{L}\end{array}$ \\
\hline \multicolumn{5}{|l|}{$C+\mu_{L}=0$} \\
\hline \multicolumn{5}{|l|}{$\begin{array}{c}0<\left(C+\mu_{L}\right)^{2} \\
<A\left(B-\mu_{L}\right)\end{array}$} \\
\hline $\begin{array}{c}\left(C+\mu_{L}\right)^{2}= \\
A\left(B-\mu_{L}\right)\end{array}$ & & & $\begin{array}{c}\left(C+\mu_{L}\right)^{2}= \\
\left(A+\mu_{T}-\mu_{L}\right) \\
\quad \cdot\left(B-\mu_{L}\right)\end{array}$ & \\
\hline $\begin{array}{c}\left(C+\mu_{L}\right)^{2}> \\
A\left(B-\mu_{L}\right)\end{array}$ & & & $\begin{array}{l}\left(C+\mu_{L}\right)^{2}> \\
\left(A+\mu_{T}-\mu_{L}\right)\end{array}$ & \\
\hline
\end{tabular}

Rays in the second mode with $\boldsymbol{\xi}$ not parallel to $\mathbf{k}$ are bounded away from the light cones for the other modes under the following conditions on the material parameters: $\mu_{L}=B \neq A+\mu_{T}$, or $A+\mu_{T} \neq \mu_{L}$ and $\mu_{L}$ lies between $A+\mu_{T}$ and $B$.

Rays in the third mode are bounded away from the light cones for the other modes under the following conditions on the material parameters:
(CM1)
$A+\mu_{T}<\mu_{L}<B$
or
$B<\mu_{L}<\mu_{T}$

Corollary 3 (Conditions for a disjoint mode along rays for $(G W P 2)$ ) Rays in the first mode with $\boldsymbol{\xi}$ not parallel to $\mathbf{k}$ are bounded away from the light cones for the other modes under the following conditions on the material parameters: $\mu_{L} \neq \mu_{T}$ and $\mu_{L}<B$.

Rays in the second mode with $\boldsymbol{\xi}$ not parallel to $\mathbf{k}$ are bounded away from the light cones for the other modes under the following conditions on the material parameters: (a) $\mu_{T} \neq \mu_{L}=B \neq A+\mu_{T}$, or (b) $\mu_{T} \neq \mu_{L}$ and $\mu_{L}<\min \left\{A+\mu_{T}, B\right\}$, or (c) $\mu_{T} \neq \mu_{L}$ and $\mu_{L}>\max \left\{A+\mu_{T}, B\right\}$.

Rays in the third mode are bounded away from the light cones for the other modes under the following conditions on the material parameters: 
Fig. 3. Types of intersection of the sheets of the slowness surface for positive-definite, transversely isotropic elastic media in families that have only one distinct $(Q 1)$, (Q2), and critical anellipticity values.

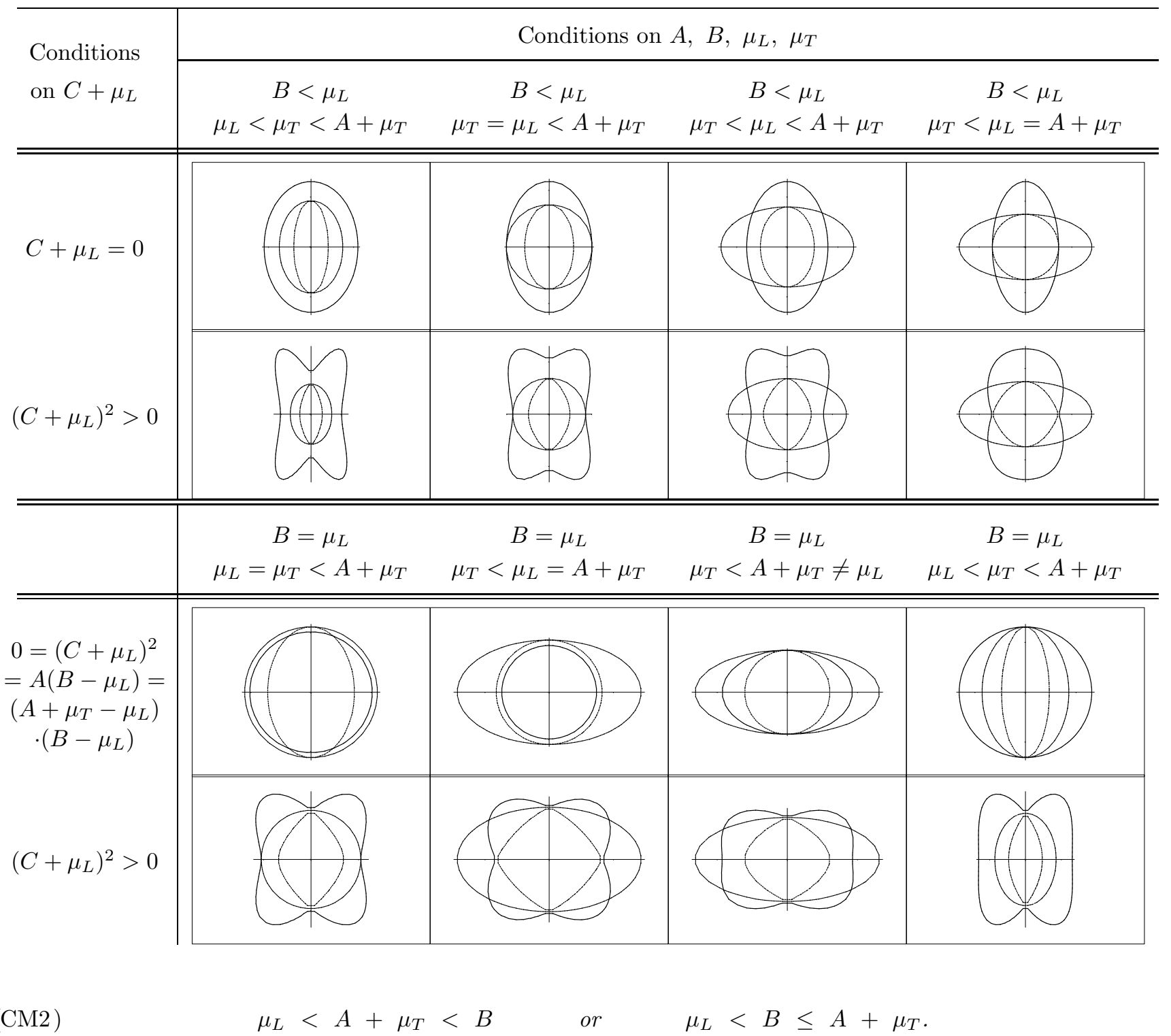

In the cases (CM1) and (CM2) of GWP with disjoint modes the only type of intersection that occurs is tangential intersection on the $\mathbf{k}$ axis, and coincident modes.

Lemma 4 (Conditions under which the sheets of the slowness surface do not intersect for GWP in strongly elliptic media) Let $1=c_{i}^{2}(\mathbf{s})$ be the sheets of the slowness surface given in Section 4 , where the $\underline{c}_{i}$ are ordered by (22) and (23).

Then, in the case (GWP1) we have $\underline{c}_{0} \neq \underline{c}_{1}$ if and only if $\boldsymbol{\xi}$ and $\mathbf{k}$ are not parallel. In addition, $\underline{c}_{1} \neq \underline{c}_{2}$ if and only if all of the following conditions hold:

(1) $\mu_{L} \neq A+\mu_{T}$ or $\mu_{L} \neq B$, i.e., the sheets for the second and third modes do not coincide; and

(2) otherwise, $\mu_{L} \neq B$ or $\boldsymbol{\xi}$ and $\mathbf{k}$ are not parallel, i.e., the sheets for the second and third modes do not intersect at the $\mathbf{k}$ axis; 
Table 1. Cases of GWP with a disjoint slowness surface

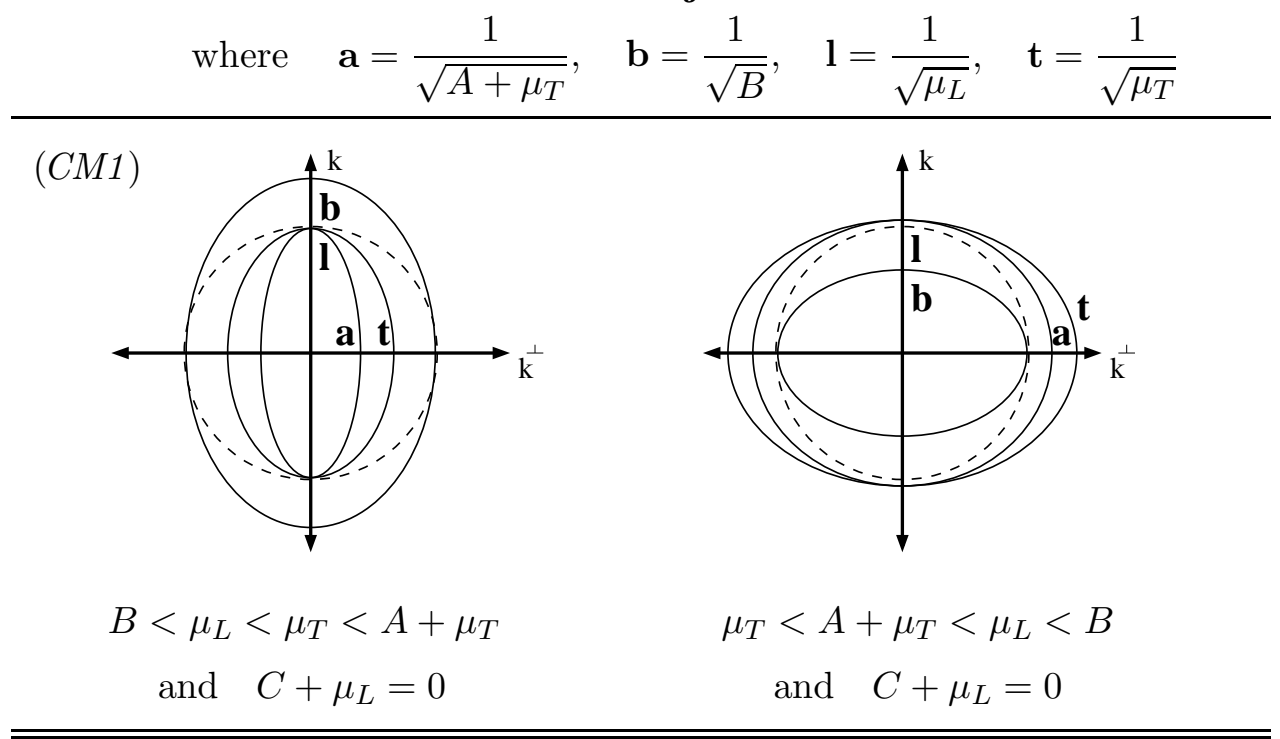

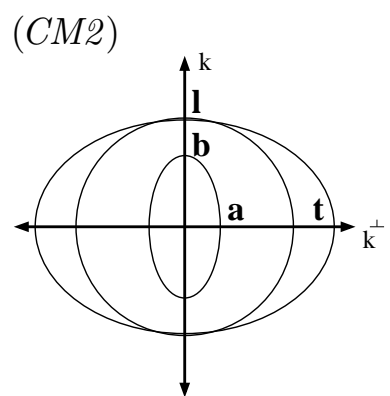

$\mu_{L}>\mu_{T}$

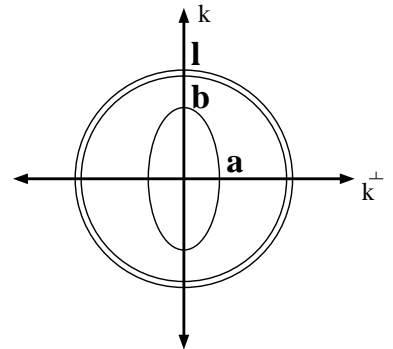

$\mu_{L}=\mu_{T}$

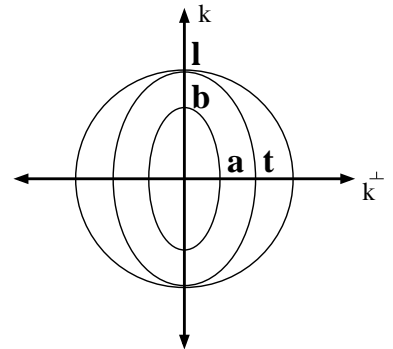

$\mu_{L}<\mu_{T}$

and $\quad \mu_{L}<\min \left\{A+\mu_{T}, B\right\}, \quad\left(C+\mu_{L}\right)^{2}=\left(A+\mu_{T}-\mu_{L}\right)\left(B-\mu_{L}\right)$

(3) and $\mu_{L} \neq A+\mu_{T}$ or $\boldsymbol{\xi}$ and $\mathbf{k}$ are not orthogonal, i.e., the sheets for the second and third modes do not intersect at an axis orthogonal to $\mathbf{k}$;

(4) and otherwise, $\mu_{L}$ lies between $A+\mu_{T}$ and $B, \mu_{L}$ agrees with one of $A+\mu_{T}$ and $B$, or

$$
\boldsymbol{\xi} \text { does not lie in the cone }\left(\mathbf{k} \cdot \frac{\boldsymbol{\xi}}{|\boldsymbol{\xi}|}\right)^{2}=\frac{A+\mu_{T}-\mu_{L}}{A+\mu_{T}+B-2 \mu_{L}},
$$

i.e., they do not intersect between their major and minor axes.

Finally, $\underline{c}_{0} \neq \underline{c}_{2}$ if and only if the same type of conditions hold, which in this case are: (1) $\mu_{L} \neq \mu_{T}$ or $\mu_{L} \neq B$; and (2) $\mu_{L} \neq B$ or $\boldsymbol{\xi}$ and $\mathbf{k}$ are not parallel; (3) $\mu_{L} \neq \mu_{T}$ or $\boldsymbol{\xi}$ and $\mathbf{k}$ are not orthogonal; and (4) $\mu_{L}$ lies between $\mu_{T}$ and $B, \mu_{L}$ agrees with one of $\mu_{T}$ and $B$, or

$$
\boldsymbol{\xi} \text { does not lie in the cone }\left(\mathbf{k} \cdot \frac{\boldsymbol{\xi}}{|\boldsymbol{\xi}|}\right)^{2}=\frac{\mu_{T}-\mu_{L}}{\mu_{T}+B-2 \mu_{L}} \text {. }
$$

In case (GWP2) we have $\underline{c}_{0} \neq \underline{c}_{1}$ if and only if $\mu_{T} \neq \mu_{L}$ and $\boldsymbol{\xi}$ and $\mathbf{k}$ are not parallel. In addition, $\underline{c}_{1} \neq \underline{c}_{2}$ if and only if all of the following conditions hold: (1) $\mu_{L} \neq A+\mu_{T}$ or $\mu_{L} \neq B$; and (2) $\mu_{L} \neq B$ or $\boldsymbol{\xi}$ and $\mathbf{k}$ are not parallel; (3) $\mu_{L} \neq A+\mu_{T}$ or $\boldsymbol{\xi}$ and $\mathbf{k}$ are not orthogonal; (4) $\mu_{L}$ is smaller than, or larger than, both of $A+\mu_{T}$ and $B, \mu_{L}$ agrees with one of $A+\mu_{T}$ and $B$, or 
Fig. 4. Types of intersection of the sheets of the slowness surface for strongly elliptic (Q1) or $($ Q2) media. The slowness surface has a rotational symmetry about the fiber direction $\mathbf{k}$, which is represented by the vertical axis. Coincident sheets are plotted slightly offset. Here we write $\alpha<\{\beta, \gamma\}$ for $\alpha<\beta$ and $\alpha<\gamma$, and $\{\alpha ; \beta=\gamma\}<\delta$ for $\alpha<\delta$ and $\beta=\gamma<\delta$.

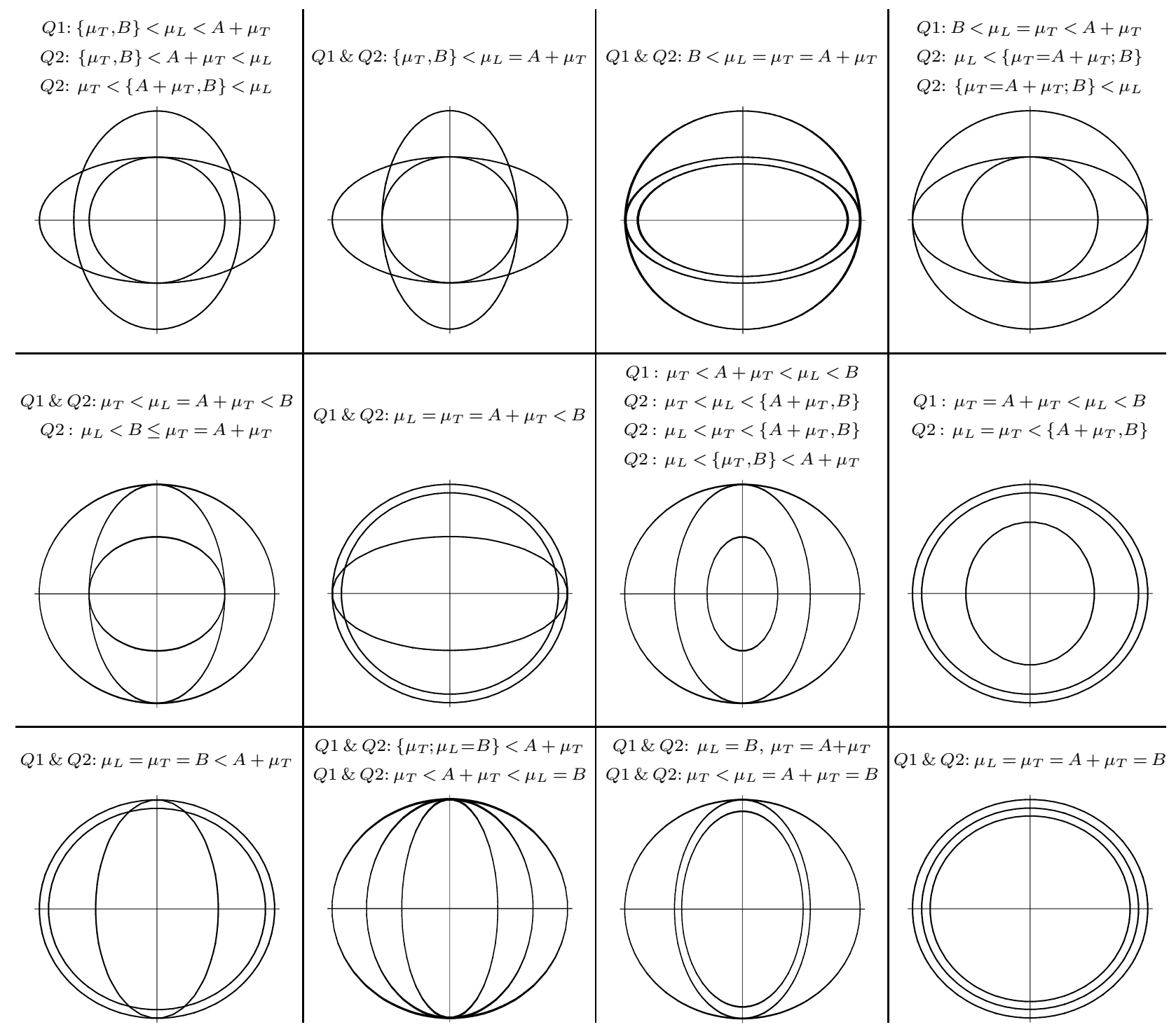

$\boldsymbol{\xi}$ does not lie in the cone $\left(\mathbf{k} \cdot \frac{\boldsymbol{\xi}}{|\boldsymbol{\xi}|}\right)^{2}=\frac{A+\mu_{T}-\mu_{L}}{A+\mu_{T}-B}$.

Finally, $\underline{c}_{0} \neq \underline{c}_{2}$ if and only if all of the following conditions hold: $\mu_{L}<B$ or

$\boldsymbol{\xi}$ does not lie in the cone $\left(\mathbf{k} \cdot \frac{\boldsymbol{\xi}}{|\boldsymbol{\xi}|}\right)^{2}=\frac{A}{\mu_{L}-B+A}$.

We prove Lemma 4 in Section 7. 
Fig. 5. Additional types of intersection of the sheets of the slowness surface for strongly elliptic $($ Q1) media. The sheets of the slowness surface are plotted slightly offset in the case of coincident modes or ridge intersections. Here we write $\alpha<\{\beta, \gamma\}$ for $\alpha<\beta$ and $\alpha<\gamma$, and $\{\alpha ; \beta=\gamma\}<\delta$ for $\alpha<\delta$ and $\beta=\gamma<\delta$.

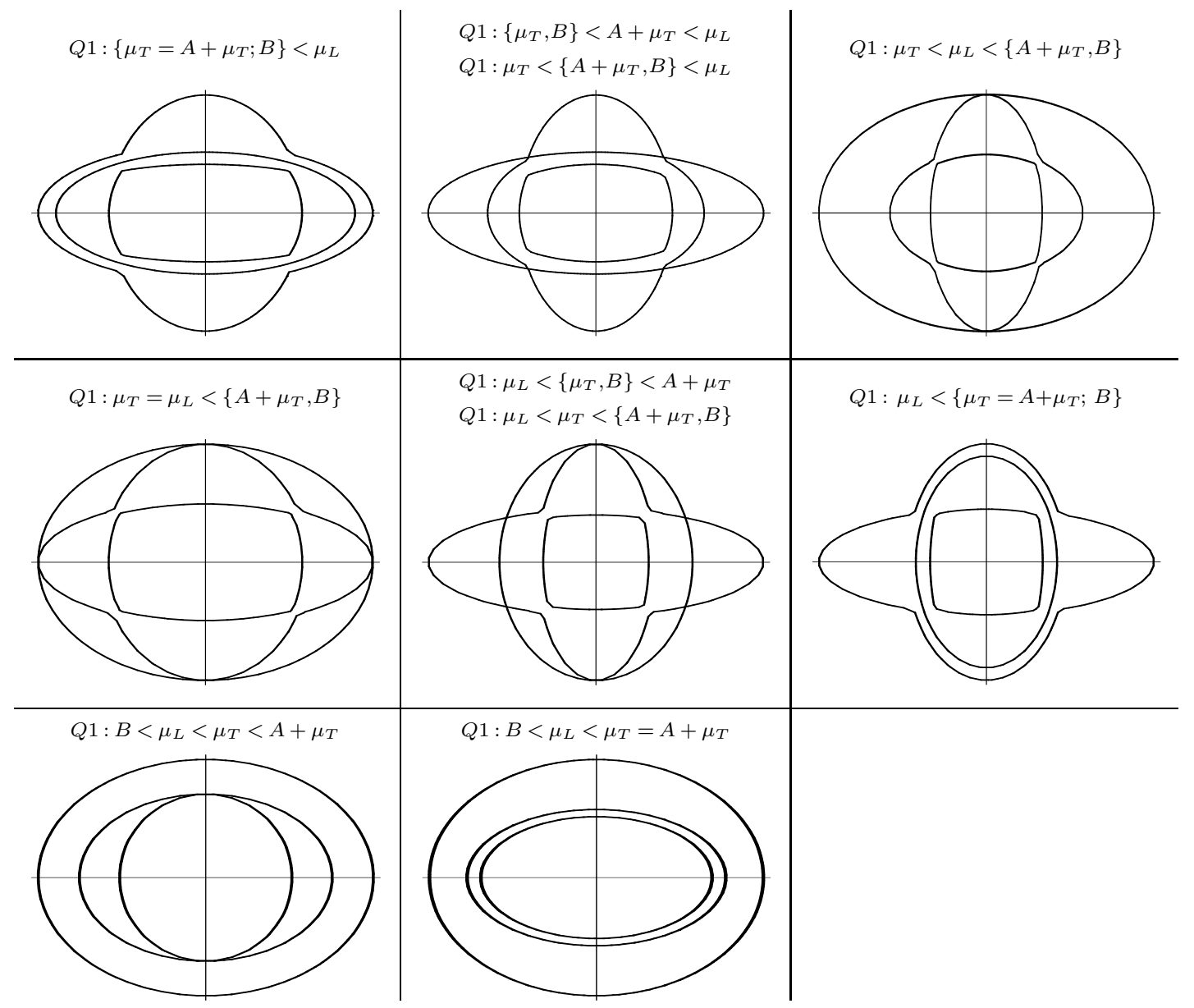

\subsection{The polarization vectors for transversely isotropic elastodynamics with GWP}

The factors $\tau^{2}-c^{2}(\boldsymbol{\xi})$ of the determinant of the principal symbol of the operator $\widehat{P}_{\rho, \mathbf{C}}$, give rise to the slowness surfaces $1=c^{2}(s)$ (and eikonal equations $\left(\partial_{t} \varphi\right)^{2}=c^{2}\left(\nabla_{x} \varphi\right)$ ). It is useful to view these factors $\tau^{2}-c^{2}(\boldsymbol{\xi})$ as eigenvalues of the principal symbol of $\widehat{P}_{\rho, \mathbf{C}}$, especially for the microlocal analysis of wave propagation.

Remark 5 (Eigenvalues of the principal symbol) The eigenvalues of the principal symbol of the operator $\widehat{P}_{\rho, \mathrm{C}}$ for elastodynamics in cases (Q1) and (Q2) are given in Section 4 by $\underline{c}^{2}(\tau, \boldsymbol{\xi})=-\tau^{2}+c^{2}$ with $c^{2}=c_{0}^{2}$ or $c^{2}=c_{ \pm}^{2}=$ $p \pm|q|$, where $c_{0}^{2}(\boldsymbol{\xi}), p(\boldsymbol{\xi})$, and $q(\boldsymbol{\xi})$ are polynomials in $\boldsymbol{\xi}$. In this case the $c_{ \pm}^{2}$ are not generally smooth functions of $\boldsymbol{\xi}$ for each $x$, (and the corresponding sheets $1=p(\mathbf{s}) \pm|q(\mathbf{s})|$ of the slowness surface are not generally ellipsoids for each $x$, although the slowness surface is a union of ellipsoids). In the case of GWP the eigenvalues of the principal symbol are given in Section 4, and are smooth functions of $x, \xi, \tau$. We order the eigenvalues as in (22) and (23) for (GWP1) and (GWP2).

The eigenvectors $\mathbf{v}_{i}$ of the principal symbol of $\widehat{P}_{\rho, \mathbf{C}}$ model the polarization vectors for each wave mode. The polarization vectors give (as a first approximation and in the case of systems of real principal type - see, for example, [24] and [25]) the direction of displacement for the wave mode. In the case of (GWP1) our presentation agrees with that of Burridge, Chadwick, and Norris $[13,(3.10)]$ when restricting to transverse isotropy, i.e., when $c_{11}=c_{22}$. In the case of 
(GWP2) our formulation is similar to that of Chadwick and Norris [12, $\left.(P 3)_{1}\right]$ since $A+\mu_{T}+C=\left(A+\mu_{T}-\mu_{L}\right)+$ $\left(C+\mu_{L}\right)=\sigma_{a}{\sqrt{\left|A+\mu_{T}-\mu_{L}\right|}}^{2}+\sigma_{c} \sqrt{\left|A+\mu_{T}-\mu_{L}\right|} \sqrt{\left|B-\mu_{L}\right|}=\sigma_{c} \sqrt{\left|A+\mu_{T}-\mu_{L}\right|} \cdot\left(\sigma_{a} \sigma_{c} \sqrt{\left|A+\mu_{T}-\mu_{L}\right|}+\right.$ $\left.\sqrt{\left|B-\mu_{L}\right|}\right)$, and similarly, $B+C=\sigma_{a} \sqrt{\left|B-\mu_{L}\right|} \cdot\left(\sigma_{a} \sigma_{c} \sqrt{\left|A+\mu_{T}-\mu_{L}\right|}+\sqrt{\left|B-\mu_{L}\right|}\right)$, where $\sigma, \sigma_{a}, \sigma_{b}, \sigma_{c}$ is the signum, respectively, of $\boldsymbol{\xi} \cdot \mathbf{k}, A+\mu_{T}-\mu_{L}, B-\mu_{L}, C+\mu_{L}$. (In particular, when the respective expression is zero, we define the corresponding $\sigma$ to be 1.) Our presentation, though, makes clear the dependence of the eigenvectors for (GWP2) on the fundamental quantities $A+\mu_{T}-\mu_{L}$ and $B-\mu_{L}$.

Proposition 6 (Eigenvectors of the principal symbol) In case (GWP1), when $\mathbf{k} \nVdash \boldsymbol{\xi}$ and the eigenvalues (22) are distinct, we have that the corresponding smooth eigenvectors with $\boldsymbol{\xi} \neq 0$ are parallel to

$$
\mathbf{v}_{0}=\mathbf{k} \wedge \boldsymbol{\xi}, \quad \mathbf{v}_{1}=-\sigma \sigma_{b} \boldsymbol{\xi}_{k^{\perp}}, \quad \mathbf{v}_{2}=\sigma \sigma_{b} \mathbf{k}
$$

In case (GWP2), when $\mathbf{k} \nVdash \boldsymbol{\xi}, A+\mu_{T} \neq \mu_{L}$, and $B \neq \mu_{L}$ (that is, (GWP1) does not hold), it follows when the eigenvectors (23) are distinct that the corresponding (mutually orthogonal) eigenvectors are parallel to

$$
\begin{aligned}
\mathbf{v}_{0}=\mathbf{k} \wedge \boldsymbol{\xi}, \quad \mathbf{v}_{1} & =-\left(\sigma_{b} \sqrt{\left|B-\mu_{L}\right|}\right) \sigma\left|\boldsymbol{\xi}_{k}\right| \frac{\boldsymbol{\xi}_{k^{\perp}}}{\left|\boldsymbol{\xi}_{k^{\perp}}\right|}+\left(\sigma_{c} \sqrt{\left|A+\mu_{T}-\mu_{L}\right|}\right)\left|\boldsymbol{\xi}_{k^{\perp}}\right| \mathbf{k} \\
\mathbf{v}_{2} & =\left(\sigma_{c} \sqrt{\left|A+\mu_{T}-\mu_{L}\right|}\right) \boldsymbol{\xi}_{k^{\perp}}+\left(\sigma_{b} \sqrt{\left|B-\mu_{L}\right|}\right) \sigma\left|\boldsymbol{\xi}_{k}\right| \mathbf{k} .
\end{aligned}
$$

If two of the eigenvalues agree (i.e., in case (GWP1), if either $\mathbf{k} \wedge \boldsymbol{\xi}=0 ; A=0 ; \quad \mu_{L}=\mu_{T}$ and $B=\mu_{L}$; $\mu_{L}=\mu_{T}$ and $\boldsymbol{\xi}_{k}=0 ; A+\mu_{T}=\mu_{L}$ and $B=\mu_{L} ;$ or $A+\mu_{T}=\mu_{L}$ and $\boldsymbol{\xi}_{k}=0 ;$ or, in case (GWP2), if either $\mathbf{k} \wedge \boldsymbol{\xi}=0 ; \mu_{L}=\mu_{T} ; A=0$ and $B=\mu_{L} ; A=0$ and $\boldsymbol{\xi}_{k}=0 ; A+\mu_{T}=\mu_{L}$ and $B=\mu_{L} ;$ or $A+\mu_{T}=\mu_{L}$ and $\left.\boldsymbol{\xi}_{k}=0\right)$, then the eigenvector corresponding to the distinct eigenvalue is given by (24), or (25), respectively, and the other two eigenvectors are restricted only in that they are orthogonal to the first. If all three eigenvalues agree, then there is no restriction on the corresponding eigenvectors.

We prove Proposition 6 in Section 7.

The quasi-compressional polarization vector is, in anisotropic media, approximately longitudinal, that is, in the direction of $\boldsymbol{\xi}$, while the quasi-shear polarization is approximately transverse to the propagating shear wave front, so is approximately orthogonal to $\boldsymbol{\xi}$. We conclude from Proposition 6 , for example, that in case $(G W P 2)$ when $A+\mu_{T} \neq \mu_{L}$, or $B \neq \mu_{L}$ and $\boldsymbol{\xi}$ is not orthogonal to $\mathbf{k}$, we have that $\mathbf{v}_{0}$ is quasi-shear, while, for example, if $\sigma, \sigma_{b}$, and $\sigma_{c}$ are all positive, then $\mathbf{v}_{1}$ is also quasi-shear, and $\mathbf{v}_{2}$ is quasi-compressional.

In particular, in case (GWP2) when $A+\mu_{T} \neq \mu_{L}$, or $B \neq \mu_{L}$ and $\boldsymbol{\xi}$ is not orthogonal to $\mathbf{k}$, we have that $\mathbf{v}_{1}(\boldsymbol{\xi})$ and $\mathbf{v}_{2}(\boldsymbol{\xi})$ lie in the $\boldsymbol{\xi}$-k-plane, are orthogonal, and form a right-handed system $\left\{\mathbf{v}_{0}, \mathbf{v}_{1}, \mathbf{v}_{2}\right\}$; also, if $A+\mu_{T}=\mu_{L}$, then $\mathbf{v}_{1}$ is always orthogonal to $\mathbf{k}$, and $\mathbf{v}_{2}$ is always parallel to $\mathbf{k}$; while if $B=\mu_{L}$ or $\boldsymbol{\xi}_{k}=0$, then $\mathbf{v}_{1}$ is always parallel to $\mathbf{k}$, while $\mathbf{v}_{2}$ is always orthogonal to $\mathbf{k}$. If $\sigma_{c} \sigma_{b}>0$, then $\mathbf{v}_{2}(\boldsymbol{\xi})$ is parallel to the direction vector of the ellipse $\left|\boldsymbol{\xi}_{k^{\perp}}\right|^{2} /\left(A+\mu_{T}-\mu_{L}\right)+\left|\boldsymbol{\xi}_{k}\right|^{2} /\left(B-\mu_{L}\right)=1$, and $\mathbf{v}_{1}$ is quasi-transverse, while $\mathbf{v}_{2}$ is quasi-longitudinal, since $\mathbf{v}_{1}(\boldsymbol{\xi}) \cdot \boldsymbol{\xi}=0$ and $\mathbf{v}_{2}(\boldsymbol{\xi}) \| \boldsymbol{\xi}$ for $\sqrt{\left|A+\mu_{T}-\mu_{L}\right|}=\sqrt{\left|B-\mu_{L}\right|}$. Otherwise, if $\sigma_{c} \sigma_{a}<0$, then $\mathbf{v}_{1}$ is a saddle vector field quasi-transverse to $\mathbf{k}$ and $(\mathrm{Span} \mathbf{k})^{\perp}$, and $\mathbf{v}_{2}$ is a saddle vector field quasi-along $\mathbf{k}$ and $(\mathrm{Span} \mathbf{k})^{\perp}$, since for $\sqrt{\left|A+\mu_{T}-\mu_{L}\right|}=\sqrt{\left|B-\mu_{L}\right|} \quad \mathbf{v}_{1}$ is a saddle vector field transverse to $\mathbf{k}$ and (Span $\left.\mathbf{k}\right)^{\perp}$, and $\mathbf{v}_{2}$ is a saddle vector field along $\mathbf{k}$ and $(\operatorname{Span} \mathbf{k})^{\perp}$.

It follows from Proposition 6 and the Implicit Function Theorem that:

Corollary 7 (Smoothness of the polarization vectors in the case of a disjoint sheet of the slowness surface) In the cases (CM1) or (CM2) the eigenvector $\mathbf{v}_{2}(\boldsymbol{\xi})$ corresponding to the disjoint mode is smooth for $\boldsymbol{\xi} \neq 0$. In addition, $\mathbf{v}_{0}$ and $\mathbf{v}_{1}$ are smooth, except on the axis of transverse isotropy $\{\boldsymbol{\xi}: \mathbf{k} \wedge \boldsymbol{\xi}=0\}$, and cannot be extended continuously there. 


\section{Proofs}

Proof of Equation (17). It follows, for example, from the proof of [25, Lemma 2.7], that the determinant of the principal symbol of $P_{\rho, \mathbf{C}}$ is given by $\left(-\rho \tau^{2}+\alpha\right)^{3}+\left(-\rho \tau^{2}+\alpha\right)^{2}\left[\beta+2 \gamma(\mathbf{k} \cdot \boldsymbol{\xi})+\delta|\boldsymbol{\xi}|^{2}\right]+\left(-\rho \tau^{2}+\alpha\right)\left(\beta \delta-\gamma^{2}\right)|\mathbf{k} \wedge \boldsymbol{\xi}|^{2}=$ $\left(-\rho \tau^{2}+\alpha\right)\left(\left(-\rho \tau^{2}+\alpha\right)^{2}+\left(-\rho \tau^{2}+\alpha\right)\left[\beta+2 \gamma(\mathbf{k} \cdot \boldsymbol{\xi})+\delta|\boldsymbol{\xi}|^{2}\right]+\left(\beta \delta-\gamma^{2}\right)\left[|\boldsymbol{\xi}|^{2}-\left|\boldsymbol{\xi}_{k}\right|^{2}\right]\right)$. The result follows by (15) and a calculation.

Proof of Proposition 1. We first square terms involved in $c_{ \pm}^{2}-c_{0}^{2}$ to find that $c_{ \pm}^{2}=c_{0}^{2}$ holds only if $\left(A\left(\mu_{T}-\right.\right.$ $\left.\left.\mu_{L}\right)\left|\boldsymbol{\xi}_{k^{\perp}}\right|^{2}-\left[A\left(B-\mu_{L}\right)-\left(C+\mu_{L}\right)^{2}\right]\left|\boldsymbol{\xi}_{k}\right|^{2}\right)\left|\boldsymbol{\xi}_{k^{\perp}}\right|^{2}=0, \quad$ in which case

$$
2 \rho\left(c_{ \pm}^{2}-c_{0}^{2}\right)=\left[A-\left(\mu_{T}-\mu_{L}\right)\right]\left|\boldsymbol{\xi}_{k^{\perp}}\right|^{2}+\left(B-\mu_{L}\right)\left|\boldsymbol{\xi}_{k}\right|^{2} \pm\left.\left|\left[A-\left(\mu_{T}-\mu_{L}\right)\right]\right| \boldsymbol{\xi}_{k^{\perp}}\right|^{2}+\left(B-\mu_{L}\right)\left|\boldsymbol{\xi}_{k}\right|^{2} \mid .
$$

At the fiber (where $\boldsymbol{\xi}_{k^{\perp}}=0$ and $\left|\boldsymbol{\xi}_{k}\right|=1$ ) we have that $2 \rho\left(c_{ \pm}^{2}-c_{0}^{2}\right)=B-\mu_{L} \pm\left|B-\mu_{L}\right|$, and so $c_{+}^{2}=c_{0}^{2}$ if and only if $B \leq \mu_{L}$, and $c_{-}^{2}=c_{0}^{2}$ if and only if $B \geq \mu_{L}$. Away from the fiber (where $\boldsymbol{\xi}_{k^{\perp}} \neq 0$ ) we have that $A\left[-\left(\mu_{T}-\mu_{L}\right)\left|\boldsymbol{\xi}_{k^{\perp}}\right|^{2}+\left(B-\mu_{L}\right)\left|\boldsymbol{\xi}_{k}\right|^{2}\right]=\left(C+\mu_{L}\right)^{2}\left|\boldsymbol{\xi}_{k}\right|^{2}$, and so for $A \neq 0$ (which holds for positive-definite elasticity tensors, since then $A>0)$ we have $2 \rho\left(c_{ \pm}^{2}-c_{0}^{2}\right)=\left[A^{2}\left|\boldsymbol{\xi}_{k^{\perp}}\right|^{2}+\left(C+\mu_{L}\right)^{2}\left|\boldsymbol{\xi}_{k}\right|^{2}\right] / A \pm\left.\left|A^{2}\right| \boldsymbol{\xi}_{k^{\perp}}\right|^{2}+\left(C+\mu_{L}\right)^{2}\left|\boldsymbol{\xi}_{k}\right|^{2}|/| A \mid$. It follows that $c_{+}^{2}=c_{0}^{2}$ does not hold, and $c_{-}^{2}=c_{0}^{2}$ if and only if $A\left(B-\mu_{L}\right)=\left(C+\mu_{L}\right)^{2}$ and $\mu_{T}=\mu_{L}$, or $A\left(B-\mu_{L}\right) \neq\left(C+\mu_{L}\right)^{2}, \quad A\left(\mu_{T}-\mu_{L}\right) /\left[A\left(B-\mu_{L}\right)-\left(C+\mu_{L}\right)^{2}\right] \geq 0, \quad$ and $\boldsymbol{\xi}$ lies on the cone $\left|\boldsymbol{\xi}_{k}\right|^{2} /\left|\boldsymbol{\xi}_{k^{\perp}}\right|^{2}=$ $A\left(\mu_{T}-\mu_{L}\right) /\left[A\left(B-\mu_{L}\right)-\left(C+\mu_{L}\right)^{2}\right]$.

In addition,

$$
\rho^{2}\left(c_{+}^{2}-c_{-}^{2}\right)^{2}=\left[\left(A+\mu_{T}-\mu_{L}\right)\left|\boldsymbol{\xi}_{k^{\perp}}\right|^{2}-\left(B-\mu_{L}\right)\left|\boldsymbol{\xi}_{k}\right|^{2}\right]^{2}+4\left(C+\mu_{L}\right)^{2}\left|\boldsymbol{\xi}_{k^{\perp}}\right|^{2}\left|\boldsymbol{\xi}_{k}\right|^{2}
$$

has roots $\left|\boldsymbol{\xi}_{k}\right|^{2}=F^{ \pm}\left|\boldsymbol{\xi}_{k^{\perp}}\right|^{2}$, where

$$
F^{ \pm}=\frac{\left(A+\mu_{T}-\mu_{L}\right)\left(B-\mu_{L}\right)-2\left(C+\mu_{L}\right)^{2} \pm 2 \sqrt{\left[\left(C+\mu_{L}\right)^{2}-\left(A+\mu_{T}-\mu_{L}\right)\left(B-\mu_{L}\right)\right]\left(C+\mu_{L}\right)^{2}}}{\left(B-\mu_{L}\right)^{2}},
$$

if $\left[\left(C+\mu_{L}\right)^{2}-\left(A+\mu_{T}-\mu_{L}\right)\left(B-\mu_{L}\right)\right]\left(C+\mu_{L}\right)^{2} \geq 0, F^{ \pm} \geq 0$, and $B \neq \mu_{L}$. Since $\left(A+\mu_{T}-\mu_{L}\right)\left(B-\mu_{L}\right) \leq\left(C+\mu_{L}\right)^{2}$ implies $F^{ \pm}<0$, it follows that $c_{+}^{2}=c_{-}^{2}$ holds in the cases given above.

Derivation of conditions $(Q 1)$ and $(Q 2)$ in Section 4. The determinant (17) of the principal symbol of $P_{\rho, \mathbf{C}}$ is of the form (3) if and only if $D^{2}$ may be written as the square of a homogeneous polynomial $p(\boldsymbol{\xi})=$ $b_{11} \xi_{1}^{2}+b_{22} \xi_{2}^{2}+b_{33} \xi_{3}^{2}+2 b_{12} \xi_{1} \xi_{2}+2 b_{13} \xi_{1} \xi_{3}+2 b_{23} \xi_{2} \xi_{3}$ in $\boldsymbol{\xi}$ of order 2. Without loss of generality we represent $p(\boldsymbol{\xi})$ as $\boldsymbol{\xi}^{t} M \boldsymbol{\xi}$, where $M(x)$ is a symmetric, $3 \times 3$ matrix.

We show now that $D^{2}$ agrees with $\left(\boldsymbol{\xi}^{t} M \boldsymbol{\xi}\right)^{2}$ if and only if $\underline{c}=0$ or $\underline{a b}=\underline{c}^{2}$, where $\underline{a}=A+\mu_{T}-\mu_{L}, \underline{b}=$ $B-\mu_{L}, \underline{c}=C+\mu_{L}$. First for any $x \in \Omega$ we write $\boldsymbol{\xi}=l(x) \mathbf{k}(x)+m(x) \mathbf{k}^{\perp}(x)+n(x) \mathbf{k}_{\perp}(x)$, where $\mathbf{k}, \mathbf{k}^{\perp}, \mathbf{k}_{\perp}$ are mutually orthonormal vectors. We conclude that $D^{2}=\underline{a}^{2}\left|\boldsymbol{\xi}_{k^{\perp}}\right|^{4}-2\left(\underline{a b}-2 \underline{c}^{2}\right)\left|\boldsymbol{\xi}_{k^{\perp}}\right|^{2}\left|\boldsymbol{\xi}_{k}\right|^{2}+\underline{b}^{2}\left|\boldsymbol{\xi}_{k}\right|^{4}$ is given by

$$
l^{4} \underline{b}^{2}+m^{4} \underline{a}^{2}+n^{4} \underline{a}^{2}-2 l^{2} m^{2}\left[\underline{a b}-2 \underline{c}^{2}\right]-2 l^{2} n^{2}\left[\underline{a b}-2 \underline{c}^{2}\right]+2 m^{2} n^{2} \underline{a}^{2} .
$$

On the other hand, a general, symmetric, $3 \times 3$ matrix

$$
M=p I+q(\mathbf{k} \otimes \mathbf{k})+r\left(\mathbf{k}^{\perp} \otimes \mathbf{k}^{\perp}\right)+2 \alpha_{11}\left(\mathbf{k}\left(s \mathbf{k}^{\perp}\right)+2 \alpha_{13}\left(\mathbf{k}\left(s \mathbf{k}_{\perp}\right)+2 \alpha_{23}\left(\mathbf{k}^{\perp}\left(\mathbf{k}_{\perp}\right)\right.\right.\right.
$$

has $\left(\boldsymbol{\xi}^{t} M \boldsymbol{\xi}\right)^{2}$ equal to $(27)$ if and only if $r=0, \alpha_{i j}=0$ for $i \neq j, p^{2}=\underline{a}^{2},(p+q)^{2}=\underline{b}^{2}, \quad M=p I+q(\mathbf{k} \otimes \mathbf{k})$, and

$$
\text { either } \quad 0=2 \underline{c}^{2}=\underline{a b}+p(p+q) \quad \text { or } \quad \underline{a b}=\underline{c}^{2}=p(p+q)
$$

The condition $\underline{c}=0$, i.e., $C+\mu_{L}=0$, is (Q1), in which case we have $p=\sigma\left(A+\mu_{T}-\mu_{L}\right)$ and $p+q=-\sigma\left(B-\mu_{L}\right)$ for $\sigma= \pm 1$; the condition $\underline{a b}=\underline{c}^{2}$, i.e.,

$$
\left(A+\mu_{T}-\mu_{L}\right)\left(B-\mu_{L}\right)=\left(C+\mu_{L}\right)^{2},
$$

is (Q2), in which case we have $p=\sigma\left(A+\mu_{T}-\mu_{L}\right)$ and $p+q=\sigma\left(B-\mu_{L}\right)$ for $\sigma= \pm 1$. 
Derivation of conditions $(\boldsymbol{G W P} 1)$ and $(\boldsymbol{G W P} 2)$ in Section 4. In case $(Q 1)$ the part $c^{2}(\boldsymbol{\xi})$ of each factor $-\rho \tau^{2}+c^{2}(\boldsymbol{\xi})$ of the determinant of the principal symbol is a polynomial in $\boldsymbol{\xi}$ if and only if the part inside the absolute value, $\left(A+\mu_{T}-\mu_{L}\right)\left|\boldsymbol{\xi}_{k^{\perp}}\right|^{2}-\left(B-\mu_{L}\right)\left|\widehat{\boldsymbol{\xi}}_{k}\right|^{2}=\left(A+\mu_{T}-\mu_{L}\right)|\boldsymbol{\xi}|^{2}-\left(A+\mu_{T}+B-2 \mu_{L}\right)\left|\widehat{\boldsymbol{\xi}}_{k}\right|^{2}=\boldsymbol{\xi}^{t} M \boldsymbol{\xi}$, is non-negative or non-positive for all $\boldsymbol{\xi}$, where $M=\left(A+\mu_{T}-\mu_{L}\right) I-\left(A+\mu_{T}+B-2 \mu_{L}\right)(\mathbf{k} \otimes \mathbf{k})$. Since $M$ has eigenvalues $A+\mu_{T}-\mu_{L}, A+\mu_{T}-\mu_{L}$, and $\mu_{L}-B$, the result follows.

In case (Q2) the condition $\left(C+\mu_{L}\right)^{2}=\left(A+\mu_{T}-\mu_{L}\right)\left(B-\mu_{L}\right)$ implies that $A+\mu_{T}-\mu_{L}$ and $B-\mu_{L}$ have the same sign. It follows that each of $c_{ \pm}^{2}(\boldsymbol{\xi})$ is a polynomial in $\boldsymbol{\xi}$ since $M=\left(A+\mu_{T}-\mu_{L}\right) I-\left(A+\mu_{T}-B\right)(\mathbf{k} \otimes \mathbf{k})$ has eigenvalues $A+\mu_{T}-\mu_{L}, A+\mu_{T}-\mu_{L}$, and $B-\mu_{L}$.

Proof of Lemma 4. In case $(G W P 1)$ the sheets of the slowness surface are ellipsoids $1=\underline{c}_{0}^{2}(\mathbf{s})=\mu_{T}\left|\mathbf{s}^{\prime}\right|^{2}+\mu_{L}(\mathbf{k}$. $s)^{2}, 1=\underline{c}_{1}^{2}(\mathbf{s})=\left(A+\mu_{T}\right)\left|\mathbf{s}^{\prime}\right|^{2}+\mu_{L}(\mathbf{k} \cdot \mathbf{s})^{2}, 1=\underline{c}_{2}^{2}(\mathbf{s})=\mu_{L}\left|\mathbf{s}^{\prime}\right|^{2}+B(\mathbf{k} \cdot \mathbf{s})^{2}$, where $\mathbf{s}^{\prime}=\mathbf{s}-(\mathbf{s} \cdot \mathbf{k}) \mathbf{k}$. It follows that $\left(\underline{c}_{1}^{2}-\underline{c}_{0}^{2}\right)(\mathbf{s})=A\left|\mathbf{s}^{\prime}\right|^{2}=0$ if and only if $\mathbf{s} \| \mathbf{k}$. Also, $\left(\underline{c}_{1}^{2}-\underline{c}_{2}^{2}\right)(\mathbf{s})=\left(A+\mu_{T}-\mu_{L}\right)\left|\mathbf{s}^{\prime}\right|^{2}+\left(\mu_{L}-B\right)(\mathbf{k} \cdot \mathbf{s})^{2}=0$ if and only if the sheets of the slowness surface coincide, or they intersect only at the $\mathbf{k}$ axis, or intersect only at an axis orthogonal to $\mathbf{k}$, or intersect only elsewhere. (See the statement of Lemma 4.) For example, they intersect only elsewhere if and only if $A+\mu_{T} \neq \mu_{L}, \mu_{L} \neq B$, and $\left(A+\mu_{T}-\mu_{L}\right)\left|\mathbf{s}^{\prime}\right|^{2}+\left(\mu_{L}-B\right)(\mathbf{k} \cdot \mathbf{s})^{2}=0$, i.e., $\left(A+\mu_{T}-\mu_{L}\right)|\mathbf{s}|^{2}+\left(2 \mu_{L}-A-\mu_{T}-B\right)(\mathbf{k} \cdot \mathbf{s})^{2}=0$, i.e., $(\mathbf{k} \cdot \mathbf{s})^{2}=\left(A+\mu_{T}-\mu_{L}\right)|\mathbf{s}|^{2} /\left(A+\mu_{T}+B-2 \mu_{L}\right)$. We can see that this last condition holds (given that $\mu_{L} \neq$ $A+\mu_{T}$ and $\left.\mu_{L} \neq B\right)$ only if $0<\left(A+\mu_{T}-\mu_{L}\right) /\left(A+\mu_{T}+B-2 \mu_{L}\right)<1$. It follows that these sheets of the slowness surface do not intersect elsewhere if and only if $\mu_{L}=A+\mu_{T}, \mu_{L}=B, 0>\left(A+\mu_{T}-\mu_{L}\right) /\left(A+\mu_{T}+B-2 \mu_{L}\right)$, $\left(A+\mu_{T}-\mu_{L}\right) /\left(A+\mu_{T}+B-2 \mu_{L}\right)>1, \quad$ or $(\mathbf{k} \cdot \mathbf{s})^{2} \neq\left(A+\mu_{T}-\mu_{L}\right)|\mathbf{s}|^{2} /\left(A+\mu_{T}+B-2 \mu_{L}\right)$. That is, $\mu_{L}=$ $A+\mu_{T}, \quad \mu_{L}=B, \quad \mu_{L}$ lies between $A+\mu_{T}$ and $\left(A+\mu_{T}+B\right) / 2, \quad \mu_{L}$ lies between $\left[2\left(A+\mu_{T}\right)+B\right] / 3$ and $B$, or $(\mathbf{k} \cdot s)^{2} \neq\left(A+\mu_{T}-\mu_{L}\right)|\mathbf{s}|^{2} /\left(A+\mu_{T}+B-2 \mu_{L}\right)$. Statement (4) in Lemma 4 for Q1 (when comparing $\underline{c}_{1}^{2}$ and $\left.\underline{c}_{2}^{2}\right)$ is established. The other cases are similar.

In case $\left(Q^{2}\right)=(G W P 2)$, the sheets of the slowness surface are the ellipsoids $1=\underline{c}_{0}^{2}(\mathbf{s})=\mu_{T}\left|s^{\prime}\right|^{2}+\mu_{L}(\mathbf{k} \cdot s)^{2}, 1=$ $\underline{c}_{1}^{2}(\mathbf{s})=\mu_{L}\left|s^{\prime}\right|^{2}+\mu_{L}(\mathbf{k} \cdot s)^{2}$, and $1=\underline{c}_{2}^{2}(\mathbf{s})=\left(A+\mu_{T}\right)\left|s^{\prime}\right|^{2}+B(\mathbf{k} \cdot s)^{2}$, where $s^{\prime}=s-(s \cdot \mathbf{k}) \mathbf{k}$, and we proceed in the same fashion.

Proof of Proposition 6. We solve the characteristic equation $\left(\sigma_{p r}(P)-\underline{c}^{2} I\right) \mathbf{v}=0$, where the principal symbol for general transverse isotropy is given by (15).

In the case that $\mathbf{k} \wedge \boldsymbol{\xi} \neq 0$ we first compute $\left(\sigma_{p r}(P)-\underline{c}^{2} I\right)(\mathbf{k} \wedge \boldsymbol{\xi}), \quad\left(\sigma_{p r}(P)-\underline{c}^{2} I\right) \boldsymbol{\xi}$, and $\left(\sigma_{p r}(P)-\underline{c}^{2} I\right) \mathbf{k}$. The resulting calculations may be summarized as:

$$
\left(\begin{array}{ccc}
\left(\sigma_{p r}(P)-\underline{c}^{2} \mathbf{I}\right) & 0 & 0 \\
0 & \left(\sigma_{p r}(P)-\underline{c}^{2} \mathbf{I}\right) & 0 \\
0 & 0 & \left(\sigma_{p r}(P)-\underline{c}^{2} \mathbf{I}\right)
\end{array}\right)\left(\begin{array}{c}
\mathbf{k} \wedge \boldsymbol{\xi} \\
\boldsymbol{\xi} \\
\mathbf{k}
\end{array}\right)=\left(\begin{array}{ccc}
\underline{E} & 0 & 0 \\
0 & \underline{A} I & \underline{B} \mathbf{I} \\
0 & \underline{C} \mathbf{I} & \underline{D} \mathbf{I}
\end{array}\right)\left(\begin{array}{c}
\mathbf{k} \wedge \boldsymbol{\xi} \\
\boldsymbol{\xi} \\
\mathbf{k}
\end{array}\right)
$$

where $\mathbf{I}$ is the $3 \times 3$ identity matrix, and

$$
\begin{aligned}
& \underline{A}=\beta(\mathbf{k} \cdot \boldsymbol{\xi})+\gamma|\boldsymbol{\xi}|^{2}=(\mathbf{k} \cdot \boldsymbol{\xi})\left(\left[-\left(A+\mu_{T}-\mu_{L}\right)+C+\mu_{L}\right]\left|\boldsymbol{\xi}_{k^{\perp}}\right|^{2}+\left[B-\mu_{L}-\left(C+\mu_{L}\right)\right]\left|\boldsymbol{\xi}_{k}\right|^{2}\right), \\
& \underline{B}=-\rho \tau^{2}+\alpha+\delta|\boldsymbol{\xi}|^{2}+\gamma(\mathbf{k} \cdot \boldsymbol{\xi})-\underline{c}^{2}=\left(A+\mu_{T}\right)\left|\boldsymbol{\xi}_{k^{\perp}}\right|^{2}+\left[\mu_{L}+C+\mu_{L}\right]\left|\boldsymbol{\xi}_{k}\right|^{2}-\underline{c}^{2}, \\
& \underline{C}=-\rho \tau^{2}+\alpha+\beta+\gamma(\mathbf{k} \cdot \boldsymbol{\xi})-\underline{c}^{2}=\mu_{L}\left|\boldsymbol{\xi}_{k^{\perp}}\right|^{2}+\left[B-\left(C+\mu_{L}\right)\right]\left|\boldsymbol{\xi}_{k}\right|^{2}-\underline{c}^{2}, \\
& \underline{D}=\gamma+\delta(\mathbf{k} \cdot \boldsymbol{\xi})=\left(C+\mu_{L}\right)(\mathbf{k} \cdot \boldsymbol{\xi}), \quad \underline{E}=-\rho \tau^{2}+\alpha-\underline{c}^{2}=\mu_{T}\left|\boldsymbol{\xi}_{k^{\perp}}\right|^{2}+\mu_{L}\left|\boldsymbol{\xi}_{k}\right|^{2}-\underline{c}^{2} .
\end{aligned}
$$

That is, if $\mathbf{k} \wedge \boldsymbol{\xi} \neq 0$, then the eigenvectors $\mathbf{v}=a(\mathbf{k} \wedge \boldsymbol{\xi})+b \boldsymbol{\xi}+c \mathbf{k}$ have components $(a, b, c)$ that satisfy

$$
\left(\begin{array}{ccc}
\underline{E} & 0 & 0 \\
0 & \underline{A} & \underline{C} \\
0 & \underline{B} & \underline{D}
\end{array}\right)\left(\begin{array}{l}
a \\
b \\
c
\end{array}\right)=\left(\begin{array}{l}
0 \\
0 \\
0
\end{array}\right)
$$

This system is solvable for $\underline{c}^{2}$ an eigenvalue of $\sigma_{p r}(P)$.

It follows in case (GWP2), for example, given the eigenvalues $\underline{c}_{i}^{2}$ in Remark 5 , that $\underline{A}=\left(\left[-\left(A+\mu_{T}-\mu_{L}\right)+\right.\right.$ 
$\left.\left.\left(C+\mu_{L}\right)\right]\left|\boldsymbol{\xi}_{k^{\perp}}\right|^{2}+\left[B-\mu_{L}-\left(C+\mu_{L}\right)\right]\left|\boldsymbol{\xi}_{k}\right|^{2}\right)(\mathbf{k} \cdot \boldsymbol{\xi}), \quad \underline{B}_{1}=-\underline{C}_{2}=\left(A+\mu_{T}-\mu_{L}\right)\left|\boldsymbol{\xi}_{k^{\perp}}\right|^{2}+\left(C+\mu_{L}\right)\left|\boldsymbol{\xi}_{k}\right|^{2}, \quad \underline{C}_{1}=$ $-\underline{B}_{2}=\left[B-\mu_{L}-\left(C+\mu_{L}\right)\right]\left|\boldsymbol{\xi}_{k}\right|^{2}, \quad \underline{D}=\left(C+\mu_{L}\right)(\mathbf{k} \cdot \boldsymbol{\xi}), \quad \underline{E}_{1}=-\left(\mu_{L}-\mu_{T}\right)\left|\boldsymbol{\xi}_{k^{\perp}}\right|^{2}, \quad \underline{E}_{2}=-A\left|\boldsymbol{\xi}_{k^{\perp}}\right|^{2}-\left(B-\mu_{L}\right)\left|\boldsymbol{\xi}_{k}\right|^{2}$. Therefore, if the three eigenvalues are distinct (i.e., $\left\{\mathbf{k} \wedge \boldsymbol{\xi} \neq 0, \mu_{L} \neq \mu_{T}, A \neq 0\right.$, and $\left.A+\mu_{T} \neq \mu_{L}\right\}$, or $\{\mathbf{k} \wedge \boldsymbol{\xi} \neq$ $0, \mu_{L} \neq \mu_{T}, B \neq \mu_{L}$, and $\left.\boldsymbol{\xi}_{k} \neq 0\right\}$ ), then $\mathbf{v}_{0}$ is parallel to $\mathbf{k} \wedge \boldsymbol{\xi}$, and the eigenvector $\mathbf{v}_{i}, i=1,2$, is a multiple of each of the following vectors that have the form $\overline{\mathbf{v}}_{i}=-\underline{D} \boldsymbol{\xi}_{k^{\perp}}+[-\underline{D}(\mathbf{k} \cdot \boldsymbol{\xi})+\underline{B}] \mathbf{k}, \widetilde{\mathbf{v}}_{i}=-\underline{C} \boldsymbol{\xi}_{k^{\perp}}+[-\underline{C}(\mathbf{k} \cdot \boldsymbol{\xi})+\underline{A}] \mathbf{k}$ :

$$
\begin{gathered}
\overline{\mathbf{v}}_{1}=\sqrt{\left|A+\mu_{T}-\mu_{L}\right|}\left(-\sigma_{c} \sqrt{\left|B-\mu_{L}\right|} \sigma\left|\boldsymbol{\xi}_{k}\right| \boldsymbol{\xi}_{k^{\perp}}+\sigma_{a} \sqrt{\left|A+\mu_{T}-\mu_{L}\right|}\left|\boldsymbol{\xi}_{k^{\perp}}\right|^{2} \mathbf{k}\right), \\
\widetilde{\mathbf{v}}_{1}=-\sigma_{c} \sigma\left|\boldsymbol{\xi}_{k}\right|\left[\sigma_{c} \sqrt{\left|A+\mu_{T}-\mu_{L}\right|}-\sigma_{b} \sqrt{\left|B-\mu_{L}\right|}\right] \\
\cdot\left(-\sigma_{c} \sqrt{\left|B-\mu_{L}\right|} \sigma\left|\boldsymbol{\xi}_{k}\right| \boldsymbol{\xi}_{k^{\perp}}+\sigma_{a} \sqrt{\left|A+\mu_{T}-\mu_{L}\right|}\left|\boldsymbol{\xi}_{k^{\perp}}\right|^{2} \mathbf{k}\right), \\
\overline{\mathbf{v}}_{2}=\sqrt{\left|B-\mu_{L}\right|} \sigma\left|\boldsymbol{\xi}_{k}\right|\left(\sigma_{c} \sqrt{\left|A+\mu_{T}-\mu_{L}\right|} \quad \boldsymbol{\xi}_{\mathbf{k}^{\perp}}+\sigma_{b} \sqrt{\left|B-\mu_{L}\right|} \sigma\left|\boldsymbol{\xi}_{k}\right| \mathbf{k}\right) \\
\widetilde{\mathbf{v}}_{2}=\sigma_{a}\left[\sigma_{c} \sqrt{\left|A+\mu_{T}-\mu_{L}\right|}\left|\boldsymbol{\xi}_{k^{\perp}}\right|^{2}+\sigma_{b} \sqrt{\left|B-\mu_{L}\right|}\left|\boldsymbol{\xi}_{k}\right|^{2}\right] \\
\cdot\left(\sigma_{c} \sqrt{\left|A+\mu_{T}-\mu_{L}\right|} \quad \boldsymbol{\xi}_{k^{\perp}}+\sigma_{b} \sqrt{\left|B-\mu_{L}\right|} \sigma\left|\boldsymbol{\xi}_{k}\right| \mathbf{k}\right) .
\end{gathered}
$$

Here again $\sigma, \sigma_{a}, \sigma_{b}, \sigma_{c}$ is the signum, respectively, of $\boldsymbol{\xi} \cdot \mathbf{k}, A+\mu_{T}-\mu_{L}, B-\mu_{L}, C+\mu_{L}$. (In particular, when the respective expression is zero, we define the corresponding $\sigma$ to be 1.) Also, we remark that $\sigma_{a}=\sigma_{b}$ in case (GWP2). The result follows.

\section{Acknowledgments}

The authors would like to acknowledge the continuous support of their Ph.D. advisers, M.E. Taylor and G. Uhlmann. They thank P. Martin and M. DeHoop for useful discussions.

\section{References}

[1] M. J. P. Musgrave, Crystal Acoustics (Holden-Day, 1970).

[2] A. L. Mazzucato and L. Rachele, On uniqueness in the inverse problem for transversely isotropic elastic media with a disjoint mode, Submitted.

[3] H. Rund, The differential geometry of Finsler spaces, in: Die Grundlehren der Mathematischen Wissenschaften, Bd. 101 (Springer-Verlag, Berlin, 1959).

[4] D. Bao, S.-S. Chern, and Z. Shen, An introduction to Riemann-Finsler geometry, volume 200 of Graduate Texts in Mathematics (Springer-Verlag, New York, 2000).

[5] P. L. Antonelli, A. Bóna, and M. A. Slawiński, Seismic rays as Finsler geodesics, Nonlinear Anal. Real World Appl. 4 (5) (2003) 711-722.

[6] M. P. Rudzki, Parametric representation of the elastic wave in anisotropic media, Journal of Applied Geophysics 54 (3-4) (2003) 165-183.

[7] K. Helbig, Foundations of anisotropy for exploration seismics (Pergamon, 1994).

[8] K. Helbig and L.Thomsen, 75-plus years of anisotropy in exploration and reservoir seismics: A historical review of concepts and methods, Geophysics 70 (6)November-December (2005) 9ND-23ND.

[9] R. G. Payton, Classification of wave front shapes for transversely isotropic elastic media, Ist. Lombardo Accad. Sci. Lett. Rend. A 108 (1974) 684-698.

[10] R. G. Payton, Elastic wave propagation in transversely isotropic media, in: Mechanics of elastic and inelastic solids, 4. Monographs and textbooks on mechanics of solids and fluids. (Kluwer Boston (ISBN 9024728436), 1983). 
[11] P. Chadwick, Wave propagation in transversely isotropic elastic media. I. Homogeneous plane waves, Proc. Roy. Soc. London Ser. A 422 (1862) (1989) 23-66.

[12] P. Chadwick and A. N. Norris, Conditions under which the slowness surface of an anisotropic elastic material is the union of aligned ellipsoids, Quart. J. Mech. Appl. Math. 43 (4) (1990) 589-603.

[13] R. Burridge, P. Chadwick, and A. N. Norris, Fundamental elastodynamic solutions for anisotropic media with ellipsoidal slowness surfaces, Proc. Roy. Soc. London Ser. A 440 (1910) (1993) 655-681.

[14] P. M. Bakker, About the completeness of the classification of cases of elliptic anistropy, Proc. Roy. Soc. London Ser. A 451 (1942) (1995) 367-373.

[15] P. F. Daley and L. R. Lines, Linearized quantities in transversely isotropic media, Can. J. Earth Sci 41 (3) (2004) 349-354.

[16] P. Theocaris and T. Philippidis, Spectral decomposition of compliance and stiffness fourth-rank tensors suitable for orthotropic materials, Z. Angew. Math. Mech. 71 (3) (1991) 161-171.

[17] A. L. Mazzucato and L. V. Rachele, Partial uniqueness and obstruction to uniqueness in inverse problems for anisotropic elastic media, J. Elasticity 83 (3) (2006) 205-245.

[18] K. H. Hellwege, H. Landolt, and R. Börnstein, Table 11, Elastic constants for the hexagonal system, and Table 13, Elastic constants for the hexagonal system (non-crystalline materials), in: Zahlenwerte und Funktionen aus Naturwissenschaften und Technik [Numerical data and functional relationships in science and technology], Neue Serie, volume III/11 (Berlin, Springer-Verlag, 1961-) 39-49.

[19] J. J. Duistermaat, Fourier integral operators (Courant Institute of Mathematical Sciences, New York University, New York, 1973).

[20] J. E. Marsden and T. J. R. Hughes, Mathematical foundations of elasticity (Dover Publications, Inc., New York, 1994).

[21] G. Duvaut and J.-L. Lions, Inequalities in mechanics and physics, volume 219 of Grundlehren der Mathematischen Wissenschaften (Springer-Verlag, Berlin, 1976).

[22] J. Merodio and R. W. Ogden, A note on strong ellipticity for transversely isotropic linearly elastic solids, Quart. J. Mech. Appl. Math. 56 (4) (2003) 589-591.

[23] P. Chadwick and A. Shuvalov, Bulk-wave polarization fields in transversely isotropic elastic media, Proceedings Of The Royal Society Of London Series A-Mathematical Physical And Engineering Sciences 453 (1956) (1997) $77-92$.

[24] N. Dencker, On the propagation of polarization sets for systems of real principal type, J. Funct. Anal. 46 (3) (1982) 351-372.

[25] L. V. Rachele, Uniqueness in inverse problems for elastic media with residual stress, Comm. Partial Differential Equations 28 (11-12) (2003) 1787-1806. 